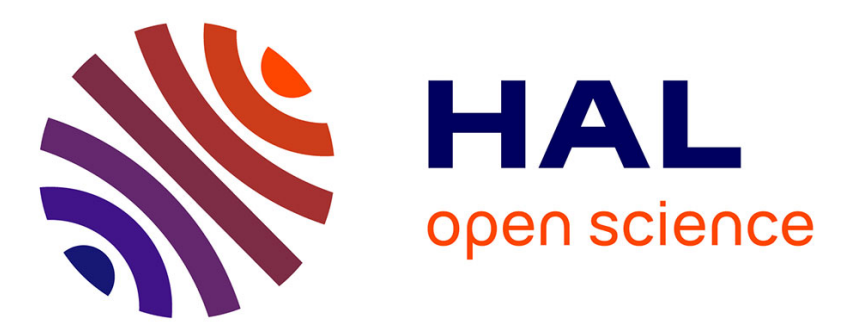

\title{
Numerical artifacts of Fast Fourier Transform solvers for elastic problems of multi-phase materials: their causes and reduction methods
}

Xiao Ma, Modesar Shakoor, Dmytro Vasiukov, Stepan Lomov, Chung Hae Park

\section{To cite this version:}

Xiao Ma, Modesar Shakoor, Dmytro Vasiukov, Stepan Lomov, Chung Hae Park. Numerical artifacts of Fast Fourier Transform solvers for elastic problems of multi-phase materials: their causes and reduction methods. Computational Mechanics, 2021. hal-03478753

\section{HAL Id: hal-03478753 \\ https://hal.science/hal-03478753}

Submitted on 14 Dec 2021

HAL is a multi-disciplinary open access archive for the deposit and dissemination of scientific research documents, whether they are published or not. The documents may come from teaching and research institutions in France or abroad, or from public or private research centers.
L'archive ouverte pluridisciplinaire HAL, est destinée au dépôt et à la diffusion de documents scientifiques de niveau recherche, publiés ou non, émanant des établissements d'enseignement et de recherche français ou étrangers, des laboratoires publics ou privés. 


\title{
Numerical artifacts of Fast Fourier Transform solvers for elastic problems of multi-phase materials: their causes and reduction methods
}

\author{
Xiao Ma ${ }^{1}$, Modesar Shakoor* ${ }^{1}$, Dmytro Vasiukov ${ }^{1}$, Stepan Lomov², and \\ Chung Hae Park ${ }^{1}$
}

${ }^{1}$ IMT Lille Douai, Institut Mines-Télécom, Univ. Lille, Centre for Materials and Processes, F-59000 Lille, France

${ }^{2}$ Department of Materials Engineering, KULeuven, Belgium

February 4, 2021

\begin{abstract}
Numerical artifacts in the form of spurious oscillations are among the critical issues of Fast Fourier Transfer (FFT) methods for solving multiphase elastic problems such as numerical homogenization, in spite of their computational simplicity and efficiency. In the first part of the present work, it is shown that the irregular discretization of the interface due to the use of a voxel-based discretization is the dominant cause of oscillations. The second part of the present work focuses on numerical artifacts reduction schemes, and in particular special treatments for dealing with the irregular discretization of the interface such as the composite voxel method and neighbor averaging methods. An improved composite voxel method by using the level-set technique is proposed, which alleviates the implementation
\end{abstract}

\footnotetext{
*Corresponding author: modesar.shakoor@imt-lille-douai.fr
} 
difficulty of the composite voxel method. This improved method is particularly relevant for non-parametrized interface representations such as those obtained from three-dimensional experimental images.

Keywords: Solids, Micromechanics, Fast Fourier Transform, Numerical artifacts, Green operator, Composite voxel method

\section{Introduction}

The Finite Element Method (FEM) is the most popular method for modeling the local mechanical behavior of composites [1]. To reproduce the smooth geometry of micro- and mesostructure of composites, a body-fitted (conformal) mesh is often used $[2,3]$. A complex fibrous geometry suffers from interpenetrations of fibres/yarns volumes, which can make building a conformal mesh impossible or lead to unacceptable quality of the mesh [4]. Improved techniques have been proposed to generate interpenetration-free conformal meshes for textile composites $[4,5]$, but they are too demanding computationally. Apart from the conforming meshes, alternative approaches exist, such as the domain superposition or embedded element technique $[6,7,8,9]$. In these approaches, the matrix and yarn domains are meshed independently, and a coupling is established between them to ensure the continuity of the displacement field.

In recent years, the pixel (2D) or voxel (3D)-based meshes are attracting more and more attention thanks to their simplicity $[10,11,12,13,14,15]$. A voxel-based mesh is a structured mesh generated directly from digital images or from a geometrical model. A box of voxels is created at first and then the voxels are labelled to be "matrix" or "fibre/yarn" depending on the position of the centroid of the voxel [16]. If the resolution of the image is too fine, such as an image output from micro-computed tomography that can easily have over billions of voxels, the computation can be too heavy for FEM. Although parallelization techniques have been proposed such as domain decomposition [17] and multigrid methods [18], their applications are not straightforward [19]. Therefore, there is a demand for approaches that would be easier to parallelize. 
Back in 1994, the Fast Fourier Transform (FFT) method was proposed initially by H.Moulinec and P.Suquet [20, 21, 22], as a voxel-based methodology that does not need stiffness matrix assembling unlike conventional FEM. In the FFT method, the local strain tensor is calculated by a convolution product with a fourth-rank Continuous Green Operator $(\mathrm{CGO})$ and a polarized term. The convolution product is transformed into a simple tensorial product in Fourier space. The calculation of the polarized term and the CGO multiplication are local operations that can be easily parallelized [19], which is also true for the FFT method in general with available packages (such as FFTW). Consequently, large-scale simulations based on full-resolution images can be performed with the FFT method. During the last decades, FFT-based methods have been applied to investigate a wide range of physical phenomena in heterogeneous media, such as eigenstrains/thermal strains [23, 24], crystal plasticity [25, 26, 27, 28, 29, 30] and damage [31, 32]. A number of physics in different types of composites have been investigated, such as the thermoelastic properties of Alumina/Al composites [33], the Kapitza interface resistance of composite conductors [34], the damage analysis of $\mathrm{SiC} / \mathrm{SiC}$ materials [19], the nonwowens [35], the effect of heterogeneous interphase on unidirectional fiber composites [36], the damage of laminates [37] and the damage analysis of textile composites [38, 39].

The FFT method proposed by H.Moulinec and P.Suquet relies on a fixed-point scheme ("basic scheme"), which consists in iteratively updating the polarized stress and reapplying the convolution with the CGO through FFTs until convergence. There are three main issues with this fixed-point scheme, which many researchers have attempted to overcome in the last two decades. The first one is the lack of convergence of the fixedpoint scheme in the case of infinite mechanical contrast between phases. An accelerated scheme was proposed in [40], with a convergence rate proportional to the square root of the contrast between the phases. Alternatives such as the "augmented Lagrangian scheme" [41] and the "polarization-based scheme" [42] have also been proposed. The second issue is that the convergence of the basic scheme is heavily sensitive to the choice of reference material. Brisard et al. [43] and Zeman [44] simultaneously proposed the use of conjugate gradient solvers as an alternative to the fixed-point scheme. An extension to the non-linear case for conjugate gradient solvers has been proposed in [45]. This solution 
appears to improve both the sensitivity to the phases contrast and the choice of reference material.

The third problem, which this paper is focused on, is the presence of spurious oscillations due to the heterogeneity of mechanical fields. One of the possible causes first reported in [22] is that the FFT method cannot satisfy Shannon's theorem for heterogeneous fields, which means that the Discrete Fourier Transform (DFT) is not equal to Fourier transform in this case. The Shannon's theorem requires that the cut-off frequency (i.e. the frequency above which the Fourier transform of the local fields vanish) is lower than half of the sampling frequency. However, a heterogeneous field in mechanics has no cut-off frequency. Another possible cause which is suggested in [46] is the Gibbs phenomenon of spectral methods. A third possible cause is proposed in [16], where the cause of oscillations that occur in voxel mesh FEM is analyzed. It is believed that these oscillations are mainly due to the non-smooth "zig-zag" interfaces between phases. The oscillations are also present when superimposed/embedded meshes are used [8, 9]. Finally, the hourglass effect, which is well known to lead to oscillations in FEM, has also been observed for the FFT method [47].

Over a decade, many researchers have worked on overcoming these artifacts. Attenuation factors with linear spline functions were proposed in [48], and low-pass filtering applied in the solutions to dampen high-frequency terms were proposed in [49]. Another strategy is to develop a consistent Discrete Green Operator (DGO) to correct the error due to the discretization of the Fourier transform. Assuming the local field is periodic, a DGO can be calculated by duplicating the local field for $k$ times in Fourier space. If $k$ is high enough, the solutions of the DGO can be approximated as those of the CGO, and refining the mesh is not required. This kind of strategy was proposed in $[43,50]$ where it was integrated into a general variational framework based on the Hashin-Strikman energy principle. Then, this strategy was applied in [46] where it was adapted to the LippmannSchwinger equation. However,in [50], the computation of the DGO was judged too costly for three-dimensional computations and a filtering approach was used instead. 
A fourth strategy is to compute the DGO based on Finite Difference (FD) discretization. This kind of approach was first reported in [51] and followed by Dreyer [52] and Brown [53] where a centered FD was considered. Backward and forward FD schemes were published by Willot in [54]. Many papers were published in the last few years in this research direction $[55,56,57,58,59]$. Among the different schemes, there is the DGO calculated based on the centered FD scheme on a rotated grid, called "rotated scheme" [60], which is one of the most interesting schemes. This scheme was implemented in the open-source software AMITEX-FFTP [61] developed by Lionel Gélébart et al. and it was successfully applied in [58] for solving field dislocation mechanics problems and in [19] for analyzing the failure of a $\mathrm{SiC} / \mathrm{SiC}$ composite. Another very interesting discretization scheme based on linear hexahedral element was proposed by Schneider et al. [62] and adapted both to the basic scheme and conjugate gradient solvers. This scheme was presented in two versions, one with reduced integration and one with full integration, similarly to FEM. It was analytically demonstrated that the scheme with reduced integration is equivalent to the "rotated scheme" proposed in [60]. These two schemes, which were proposed in [60] and in [62], will be discussed in this paper.

As illustrated in Fig. 1, the oscillations are also present even with the rotated scheme. They can be due to a non-smooth interface [16]. Remedies as "composite voxels" [63, 64] or "neighbour voxels average" [65] were proposed and applied to FFT solvers.

The performance of the different algorithms is often assessed only with respect to the macroscopic responses. When dealing with damage analysis, local response is of significant importance (interface response in particular), of which quantitative comparisons between FFT and FEM are rarely reported. The impacts and relative effects of different causes of oscillations are rarely discussed. There is no assessment of the efficiency of the composite voxel and neighbour voxels average methods in dealing with these particular issues.

Therefore, the purposes of this paper are as follows: 1) Identifying the causes of spurious oscillations in FFT and evaluating their impacts; 2) Using the results of FEM as 


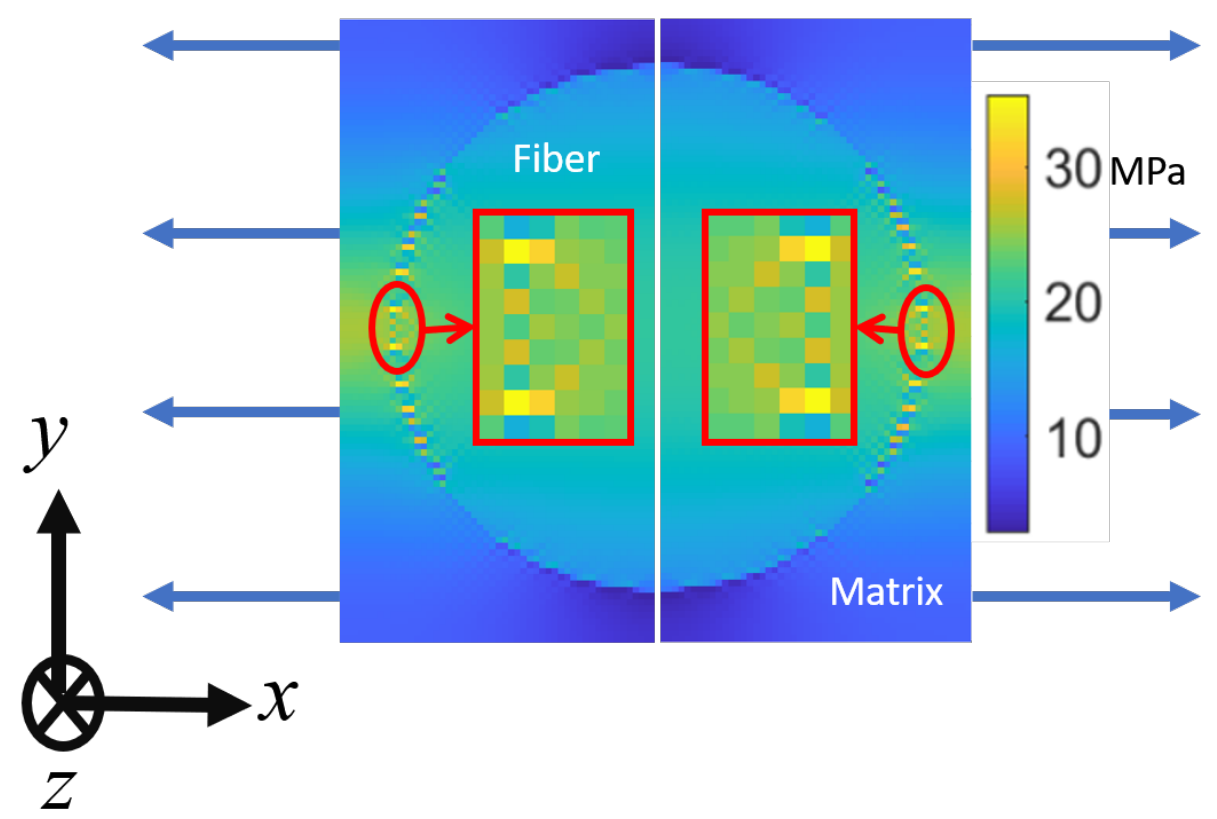

Figure 1: Illustration of spurious oscillations (check-board patterns) of local stress field $\left(\sigma_{x x}\right)$ present in FFT with rotated scheme proposed in [60] (left half part) and FEM (right half part) under tension in $x$-direction with the contrast between the fiber and matrix Young's modulii set to $E_{f} / E_{m}=22$

the reference and comparing quantitatively the influence of different Green operators (proposed in $[21,60,62]$ ) on different models and concluding on an optimal choice; 3) Proposing an improved composite voxel method and comparing it with the neighbour voxels average technique.

The paper is organized as follows: In Section 2, the different FFT based algorithms used in this paper will be presented; In Section 3, the composite voxel and neighbour voxels average methods will be discussed and an improved composite voxel method will be proposed; In Section 4, three benchmark models will be presented and used to assess different causes of oscillations in local responses and compare the numerical methods; Section 5 will be the general conclusions. 


\section{FFT methods for composites homogenization}

In this section, a review of the FFT based numerical method proposed in $[20,21,22]$ is detailed as well as some recent improved schemes [60,62] for reduction of the spurious oscillations, which will be applied and discussed in the next sections.

\subsection{The basic scheme of the FFT method and the Lippmann- Schwinger equation}

In this paper, the simulation domain is a periodic unit-cell $V$ composed of different phases that are assumed to be perfectly bonded. This unit-cell is subjected to a uniform overall strain denoted $\langle\epsilon\rangle$. This uniform overall strain is equivalent to the spatial average of strain field $\epsilon(x)$ over the domain. Displacement, strain, and Cauchy stress fields are denoted $u$, $\epsilon$ and $\sigma$ respectively. Note that the local strain $\epsilon(x)$ and the displacement $u(x)$ fields can be split into the spatial average and a fluctuation terms, which are expressed as follows:

$$
\epsilon(x)=\epsilon^{*}(x)+\langle\epsilon\rangle \quad \text { or } \quad u(x)=u^{*}(x)+\langle\epsilon\rangle \cdot x
$$

where $\epsilon^{*}(x)$ and $u^{*}(x)$ are the local fluctuations of strain and displacement fields respectively with $u^{*}(x)$ being periodic. Defining the local stiffness tensor as $C(x)$ and introducing a reference material with stiffness $C_{0}$, Hooke's law for linear elasticity is written as:

$$
\sigma(x)=C_{0}: \epsilon(x)+\tau(x), \quad \forall x \in V
$$

where $\tau(x)=\left(C(x)-C_{0}\right): \epsilon(x)$. It can be shown [20] that the equilibrium equation

$$
\operatorname{div}(\sigma(x))=0, \quad \forall x \in V
$$

with the constitutive equation in Eq. (2) is equivalent to the periodic Lippmann-Schwinger equation

$$
\epsilon(x)=-\Gamma_{0}(x) * \tau(x)+\langle\epsilon\rangle, \quad \forall x \in V
$$

where $*$ denotes a convolution product and $\Gamma_{0}$ is the fourth-order tensor field that is called Green operator. Meanwhile, the strain $\epsilon(x)$ should satisfy the compatibility condition, which is:

$$
\epsilon(x)=\frac{1}{2}\left(\nabla u(x)+(\nabla u(x))^{T}\right)
$$


Solving Eq. (4) is not an easy task in real space because the numerical computation of a convolution product is complicated, and furthermore, the term $\Gamma_{0}$ is easier to calculate Fourier space. Thus, the Eq. (4) can be easill written in Fourier space as follows:

$$
\widehat{\epsilon}(\xi)=-\widehat{\Gamma}_{0}(\xi): \widehat{\tau}(\xi), \quad \forall \xi \neq 0, \widehat{\epsilon}(0)=\langle\epsilon\rangle
$$

where the convolution can be transformed into a simple double contraction (denoted as :). Here $\widehat{\epsilon}, \widehat{\Gamma}_{0}$ and $\widehat{\tau}$ are the Fourier transforms of $\epsilon, \Gamma_{0}$ and $\tau$ respectively. The vector of frequency, denoted by $\xi$, varies in Fourier space.

Because the polarization stress term $\tau(x)$ in Eq. (2) is nonlinear, an iterative algorithm is necessary. The principle of the algorithm is to use an iterative fixed-point scheme to solve Eq. (2) and Eq. (3):

Initialization:

$\left(a_{0}\right)$

$\left(b_{0}\right)$

Iteration $(i+1)$ :

$$
\begin{gathered}
\epsilon^{0}(x)=\langle\epsilon\rangle, \quad \forall x \in V \\
\sigma^{0}(x)=C(x): \epsilon^{0}(x)
\end{gathered}
$$

$$
\epsilon^{i} \text { and } \sigma^{i} \text { are known }
$$

$$
\tau^{i}=\sigma^{i}-C_{0}: \epsilon^{i}(x)
$$

$$
\widehat{\tau}^{i}=F\left(\tau^{i}\right)
$$

$$
\begin{gathered}
\widehat{\epsilon}^{i+1}(\xi)=-\widehat{\Gamma}_{0}(\xi): \widehat{\tau}^{i}(\xi), \quad \forall \xi \neq 0, \widehat{\epsilon}(0)=\langle\epsilon\rangle \\
\epsilon^{i+1}=F^{-1}\left(\widehat{\epsilon}^{i+1}\right) \\
\sigma^{i+1}(x)=C(x): \epsilon^{i+1}(x)
\end{gathered}
$$

Convergence test: $\frac{\left(\left\langle\| \operatorname{div}\left(\sigma^{i+1} \|\right\rangle^{2}\right)^{1 / 2}\right.}{\left\|\left\langle\sigma^{i+1}\right\rangle\right\|} \leq e$

Here $\|$.$\| is the L^{2}$ norm. The iterative algorithm (6) will be stopped when the convergence test is satisfied ( $e=1 \times 10^{-12}$ in our calculations). 
In numerical calculation, the DFT is used. The unit cell is discretized into a regular grid of $N_{1} \times N_{2}$ pixels in 2D cases and $N_{1} \times N_{2} \times N_{3}$ voxels in 3D problems. For 3D cases, the coordinates of each voxel labeled by $i_{1}, i_{2}$, and $i_{3}$ are

$$
x\left(i_{1}, i_{2}, i_{3}\right)=\left(\left(i_{1}-\frac{1}{2}\right) \frac{T_{1}}{N_{1}},\left(i_{2}-\frac{1}{2}\right) \frac{T_{2}}{N_{2}},\left(i_{3}-\frac{1}{2}\right) \frac{T_{3}}{N_{3}}\right)
$$

with

$$
i_{1}=1 \ldots N_{1}, i_{2}=1 \ldots N_{2}, i_{3}=1 \ldots N_{3}
$$

and $T_{i}$ is the period of the unit cell in $i^{\text {th }}$ direction. The definition of the discrete frequencies $\xi_{i}$ depends on whether $N_{i}$ is even or odd. If $N_{i}$ is even, the discrete frequencies are given by:

$$
\xi_{i}=\left(-\frac{N_{i}}{2}+1\right) \frac{1}{T_{i}},\left(-\frac{N_{i}}{2}+2\right) \frac{1}{T_{i}}, \ldots,-\frac{1}{T_{i}}, 0, \frac{1}{T_{i}}, \ldots,\left(\frac{N_{i}}{2}-1\right) \frac{1}{T_{i}},\left(\frac{N_{i}}{2}\right) \frac{1}{T_{i}}
$$

and if $N_{i}$ is odd:

$$
\xi_{i}=-\frac{N_{i}-1}{2} \frac{1}{T_{i}}, \ldots,-\frac{1}{T_{i}}, \frac{1}{T_{i}}, \ldots, \frac{N_{i}-1}{2} \frac{1}{T_{i}} .
$$

The $\xi$ and $x$ in Alg. (6) should be discretized as a DFT is used in practice. However, as shown in $[22,60]$, when $N_{i}$ is even, the $\widehat{\Gamma}_{0}^{*}(\xi)$ (complex conjugate of $\widehat{\Gamma}_{0}(\xi)$ ) is not equal to $\widehat{\Gamma}_{0}(\xi)$ when one of the components of $\xi$ is equal to the highest frequency. Therefore, the inverse of the Fourier transform of $\widehat{\Gamma}_{0}(\xi): \widehat{\tau}(\xi)$ at step (d) of Alg. (6) has a nonzero imaginary part. To overcome this problem, $\widehat{\Gamma}_{0}(\xi)=\left(C_{0}\right)^{-1}$ may be enforced when $\xi=\left(\frac{N_{i}}{2}\right) \frac{1}{T_{i}}$. To avoid this, $N_{i}$ can be chosen odd, as done in all our calculations.

\subsection{The Green operator of Moulinec-Suquet $\left(\widehat{\Gamma}_{0}^{M-S}\right)$}

Accompanied by the proposition of FFT methods, the expression of the Green operator is also given in [21] and detailed in [22]. Following the equations (1), (2), (3) and (5), the difficulty of finding the expression of $\widehat{\Gamma}_{0}^{M-S}(\xi)$ depends on the choice of reference material. In most studies, even for anisotropic materials, an isotropic reference material is chosen. In this case, $\widehat{\Gamma}_{0}^{M-S}(\xi)$ is

$$
\widehat{\Gamma}_{0, i j k l}^{M-S}(\xi)=\frac{\left(\delta_{k i} \xi_{l} \xi_{j}+\delta_{l i} \xi_{k} \xi_{j}+\delta_{k j} \xi_{l} \xi_{i}+\delta_{l j} \xi_{k} \xi_{i}\right)}{4 \mu_{0}\|\xi\|^{2}}-\frac{\lambda_{0}+\mu_{0}}{\mu_{0}\left(\lambda_{0}+2 \mu_{0}\right)} \frac{\xi_{i} \xi_{j} \xi_{k} \xi_{l}}{\|\xi\|^{4}}
$$


where $\mu^{0}$ and $\lambda^{0}$ are the Lamé coefficients of the reference material. The Dirac delta function is denoted by $\delta$. Eq.(7) is valid only if $\xi \neq 0$. Otherwise,

$$
\widehat{\Gamma}_{0}^{M-S}(0)=0
$$

Because the Eqs. (3) and (5) are continuous, Eq. (7) is also continuous. In numerical calculations, the domain is discretized by voxels, thus the DFT is applied and the local fields $u, \epsilon$ and $\sigma$ are always calculated at the centroid of each voxel. In the DFT, $\xi$ is discretized in limited series where the high frequencies are ignored. Therefore, the Green operator of Moulinec-Suquet can also be called as "truncated Green operator".

\subsection{Willot's rotated scheme}

Inspired with the FD technique, Willot [60] modifyied the equilibrium equation and strain compatibility in Eqs. (3) and (5) from a continuous form into discrete form. They are given as follows:

$$
\widehat{k}_{i}^{*}(\xi) \widehat{\sigma}_{i j}(\xi)=0, \quad \widehat{\epsilon}_{i j}=\frac{1}{2}\left[\widehat{k}_{i}(\xi) \widehat{u}_{j}(\xi)+\widehat{k}_{j}(\xi) \widehat{u}_{i}(\xi)\right]
$$

where $\widehat{k}^{*}$ and $\widehat{k}$ represent the discrete divergence and gradient operators. Based on the Eq. (9), the DGO $\widehat{\Gamma}_{0}^{W R}$ of Willot's rotated scheme is

$$
\widehat{\Gamma}_{0, i j k l}^{W R}=\left\{\widehat{k}_{i}(\xi)\left[\widehat{k}_{m}(\xi) C_{0, m j k n} \widehat{k}_{n}^{*}(\xi)\right]^{-1} \widehat{k}_{l}^{*}(\xi)\right\}_{\text {sym }}
$$

where the symbol ${ }_{\text {sym }}$ indicates the minor symmetrization with respect to the index $(i, j)$ and $(k, l)$. As stated previously, this definition is only valid for non-zero frequency vectors so that Eq.(8) still applies. In Willot's rotated scheme, the displacement field $u$ and the divergence of the stress field are evaluated at the corners of each voxel, while the strain $\epsilon$ and the stress $\sigma$ fields lie at the centers. Based on the paper [60], for 3D cases, the $\widehat{k}_{i}$ of the rotated scheme (denoted $\widehat{k}_{i}^{R}$ ) are given as follows:

$$
\widehat{k}_{i}^{R}=\frac{J}{4} \tan \left(\frac{2 \pi \xi_{i}}{2}\right)\left(1+e^{J 2 \pi \xi_{1}}\right)\left(1+e^{J 2 \pi \xi_{2}}\right)\left(1+e^{J 2 \pi \xi_{3}}\right)
$$

where the symbol $J=\sqrt{-1}$. With the assumption of isotropic reference material, the full version of the Green operator of Willot's rotated scheme $\widehat{\Gamma}_{0}^{W R}$ in the $3 \mathrm{D}$ case can be given as:

$$
\widehat{\Gamma}_{0, i j k l}^{W R}=\frac{\left(\lambda_{0}+2 \mu_{0}\right)\left(r_{i} r_{l}^{*} \delta_{j k}\right)_{s y m}+\lambda_{0}\left[\left(r_{i} r_{l}^{*} s_{j k}\right)_{s y m}-R e\left(r_{i} r_{j}^{*}\right) \operatorname{Re}\left(r_{k} r_{l}^{*}\right)\right]-\mu_{0} r_{i} r_{j} r_{k}^{*} r_{l}^{*}}{\mu_{0}\left[2\left(\lambda_{)}+\mu_{0}\right)-\lambda_{0} \| r_{1}^{2}+r_{2}^{2}+r_{3}^{2}\right] \|^{2}}
$$


where $r_{i}=\widehat{k}_{i} /\|\widehat{k}\|$ and $s$ is the symmetric second-order tensor:

$$
s_{j k}=-4 \operatorname{Im}\left(r_{k} r_{j}^{*}\right) \operatorname{Im}\left(r_{k} r_{i}^{*}\right), i \neq j \neq k \neq l, s_{j j}=4 \operatorname{Im}\left(r_{i} r_{k}^{*}\right)^{2} .
$$

Here $\operatorname{Re}($.$) and \operatorname{Im}($.$) are the real part and the imaginary part of complex values respec-$ tively.

\subsection{Schneider's hex scheme}

The work of Willot [60] is interesting not only for the new DGO based on the rotated scheme but also for the comparison between various finite difference schemes and how they lead to various discrete Green operators. Based on this work, Schneider and coauthors [62] proposed to use the finite element method instead of the finite difference method to construct the DGO. They compared two types of finite elements, the classical trilinear hexahedral element with full integration and the one with reduced integration.

Following the notations of [62], it is reminded that the coordinates of the integration points for the reference fully integrated hexahedral element $[0,1]^{3}$ are

$$
\begin{gathered}
z_{\beta}=\left(a_{\beta_{1}}, a_{\beta_{2}}, a_{\beta_{3}}\right), \beta \in\{0,1\}^{3} \\
\text { with } a_{0}=\frac{3-\sqrt{3}}{6}, a_{1}=\frac{3+\sqrt{3}}{6},
\end{gathered}
$$

and the finite element basis functions are of the form

$$
\begin{gathered}
\prod_{i=1}^{3} h_{\alpha_{i}}\left(z_{i}\right), \alpha \in\{0,1\}^{3}, z \in[0,1]^{3} \\
\text { with } h_{0}(a)=1-a, h_{1}(a)=a, a \in[0,1] .
\end{gathered}
$$

Similarly to Eq.(2.3), the DGO for hexahedral elements is

$$
\widehat{\Gamma}_{0}^{S}=B_{K}\left(B_{K}^{H} M_{0} B_{K}\right)^{-1} B_{K}^{H}
$$

where $B_{K}$ is the discrete symmetric gradient operator and $-B_{K}^{H}$ is the discrete divergence operator at voxel $K$. Note that $B_{K}^{H}$ is the Hermitian conjugate (the transpose of the complex conjugate) of $B_{K}$. The operator $M_{0}$ is equal to $C_{0}$ when using reduced integration. Otherwise, $M_{0} \in \mathbb{R}^{8 \times 3 \times 3 \times 8 \times 3 \times 3}$ is defined as $C_{0}$ applied independently at each integration point

$$
M_{0, \alpha i j \beta k l}=\left\{\begin{array}{l}
C_{0, i j k l}, \alpha=\beta \\
0, \alpha \neq \beta
\end{array} \quad, \alpha, \beta \in\{0,1\}^{3}, i, j, k, l=1 \ldots 3\right.
$$


The formula for the discrete symmetric gradient operator $B_{K}$ is similar to that in Eq.(9), but this symmetric gradient is now computed at each integration point $\beta$ of each voxel $K$ and thus involves a gradient operator $\hat{k}_{K, \beta}$. There are always eight symmetric gradient operators $\tilde{B}_{K, \beta} \in \mathbb{C}^{3 \times 3 \times 3}$ to compute per voxel:

- in full integration, $B_{K}=\left(\tilde{B}_{K, \beta}\right)_{\beta \in\{0,1\}^{3}} \in \mathbb{C}^{8 \times 3 \times 3 \times 3}$ and $B_{K}^{H} \in \mathbb{C}^{3 \times 3 \times 3 \times 8}$,

- in reduced integration, $B_{K}=\frac{1}{8} \sum_{\beta \in\{0,1\}^{3}} \tilde{B}_{K, \beta} \in \mathbb{C}^{3 \times 3 \times 3}$ and $B_{K}^{H} \in \mathbb{C}^{3 \times 3 \times 3}$.

As a consequence, we have

$$
\tilde{B}_{K, \beta i j k}=\frac{1}{2}\left[\widehat{k}_{K, \beta i}(\xi) \delta_{j k}+\widehat{k}_{K, \beta j}(\xi) \delta_{i k}\right] .
$$

The discrete gradient operator $\widehat{k}_{K, \beta}(\xi) \in \mathbb{C}^{3}$ is given by

$$
\widehat{k}_{K, \beta, i}(\xi)=\sum_{\alpha \in\{0,1\}^{3}}\left(\begin{array}{c}
\exp \left(-2 \pi J \sum_{j=1}^{3} \frac{\xi_{j} \alpha_{j}}{N_{j}}\right)(-1)^{\alpha_{i}+1} \prod_{k} h_{\alpha_{k}}\left(z_{\beta_{k}}\right) \\
k \neq i \\
k \neq i
\end{array}\right)
$$

with satisfying Eq. (8).

To summarize, the basic scheme of the FFT method has been detailed as well as its original Green operator proposed by Moulinec et Suquet in [20, 21, 22]. Furthermore, two improved schemes, Willot's rotated scheme and Schneider's hex scheme, have been presented. They improve the procedure of Green operator calculation to reduce the spurious oscillations. It has been proven in [62] that the hex scheme of Schneider with reduced integration is equivalent to the rotated scheme of Willot. It is reminded that reduced integration is well known to produce numerical artifacts known as the hourglass effect in FEM computations. A similar difference between reduced and full integration when using FFT based numerical methods has been reported in [47].

\section{$3 \quad$ Numerical model treatment and post-treatment}

As discussed in Section 1, based on our research, the oscillations persist even though a DGO is used, which could be due to the non-smooth interface. Therefore, in this section, 
the composite voxel method as well as our improvement and the neighbour voxels average method are presented, which are believed to deal with this issue.

\subsection{Composite voxel method}

In a voxel mesh, the properties of each voxel are usually equal to the properties of the material which is present at the center of the voxel. It is proper if the total volume of a voxel belongs to only one material. However, in the case of voxels shared by several phases and thus crossed by one or multiple interfaces, this kind of properties assignment is a rough approximation and zig-zag interfaces are formed (shown in Fig. 2(a)). It is one of the reasons that limit the application of voxel-based meshes because interfaces are not as smooth as in a conformal mesh and are far from reality. More importantly, the zig-zag interface is one of the important causes of oscillations that occur near the interface. These oscillations are produced both in FFT methods and the FEM as long as a voxel mesh is used. In consequence, the composite voxel method is introduced to smooth the interface.

\subsubsection{Conventional composite voxel method}

The composite voxel approach was proposed by Kabel et al. in [63]. The properties of voxels crossed by an interface are evaluated by a local homogenization based on the properties of phases inside the voxel and their volume fraction (shown in Fig. 2(b)). Denoting the outgoing normal vector of the interface between the dominant phase and the other phases within the voxel as $n$, the formula for calculating the elastic tensor of composite voxel given by Kabel et al. in [63] is

$$
\left(P+K\left(C_{\text {laminate }}-K I d\right)^{-1}\right)^{-1}=\left\langle\left(P+K(C-K I d)^{-1}\right)^{-1}\right\rangle
$$

where $C_{\text {laminate }}$ is the homogenized elastic tensor of a composite voxel and $K>0$ is a factor that should be chosen sufficiently large. According to [63], $K$ should be larger than

the largest eigenvalue of the stiffness matrices $C(x)$ for all $x$ in $V$. The identity matrix is denoted $I d$ and $P$ is the fourth-order tensor that depends on the normal vector $n$ as follows:

$$
P_{i j k l}=\frac{1}{2}\left(n_{i} \delta_{j k} n_{l}+n_{i} \delta_{j l} n_{k}+n_{j} \delta_{i k} n_{l}+n_{j} \delta i l n_{k}\right)-n_{i} n_{j} n_{l} n_{m} .
$$


For calculating the volume fraction of each constituent in a composite voxel, one of the classical methods, called "sub-voxel approximation", is proposed in [63]. As shown in Fig. 2(b,c), at first, the composite voxel should be divided by the number of sub-voxels $N_{\text {sub }}$. Then the center position of each sub-voxel $\left(x_{\text {sub }}^{i}, y_{\text {sub }}^{i}, z_{\text {sub }}^{i}\right)$ is calculated and the constituent $\left(p h^{i}\right)$ at that position is chosen. Next, the properties of the chosen constituent $\left(C_{p h^{i}}\right)$ are assigned as the properties of the sub-voxel. The number of sub-voxels with the same constituent $\left(N_{s u b}^{p h^{i}}\right)$ divided by the total sub-voxel number inside the composite voxel is the volume fraction of this constituent in a composite voxel $\left(V_{f}^{p h^{i}}=N_{s u b}^{p h^{i}} / N_{s u b}\right)$.

To calculate the normal vector of a composite voxel, one method proposed in [63] is connecting the center of mass of the dominant material with the center of the composite voxel. More precisely, assuming $W$ to be the composite voxel and $S \subset W$ to be the dominant phase, the formula of the normal vector is given as follows:

$$
\tilde{n}:=\frac{1}{|S|} \int_{S} x d x-\frac{1}{|W|} \int_{W} x d x, n=\frac{\tilde{n}}{\|\tilde{n}\|}
$$

where $|w|=\int_{W} d x$ and $|S|=\int_{S} d x$. In numerical calculations, Eq. (12) can be expressed as:

$$
\tilde{n}:=\frac{1}{N_{\text {sub }}^{S}} \sum\left(x_{\text {sub }}^{S}, y_{\text {sub }}^{S}, z_{\text {sub }}^{S}\right)-\left(x_{0}, y_{0}, z_{0}\right)
$$

where $\left(x_{0}, y_{0}, z_{0}\right)$ is the center of the composite voxel.

\subsubsection{Improved composite voxel method}

The conventional composite voxel has a big issue: a parametric representation of the interface and its geometry should be known in advance. When extracting a model directly from an image obtained using for instance scanning electron microscopy, where the real interfaces are not clear and only the zig-zag interfaces can be determined, the conventional composite voxel can have problems. Besides, for some complex structures (e.g. textile composite and short fibers composite), it is also difficult to describe the interface mathematically. For a composite voxel containing two or more phases, an approach mainly inspired from the level-set method is proposed hereafter to calculate the volume fraction of a phase and the normal vector in a composite voxel. 


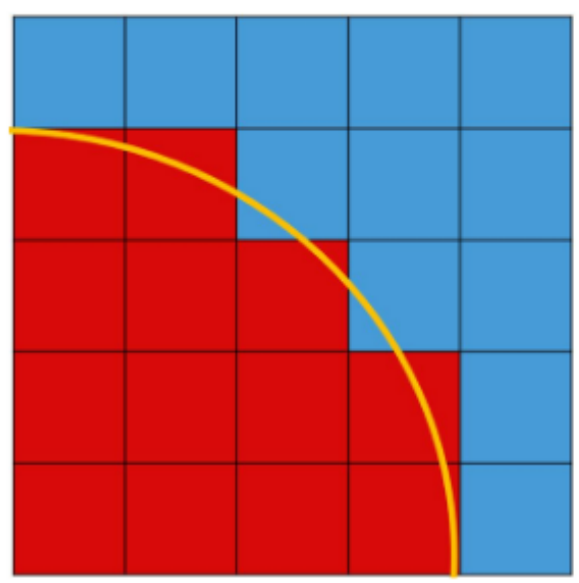

(a)

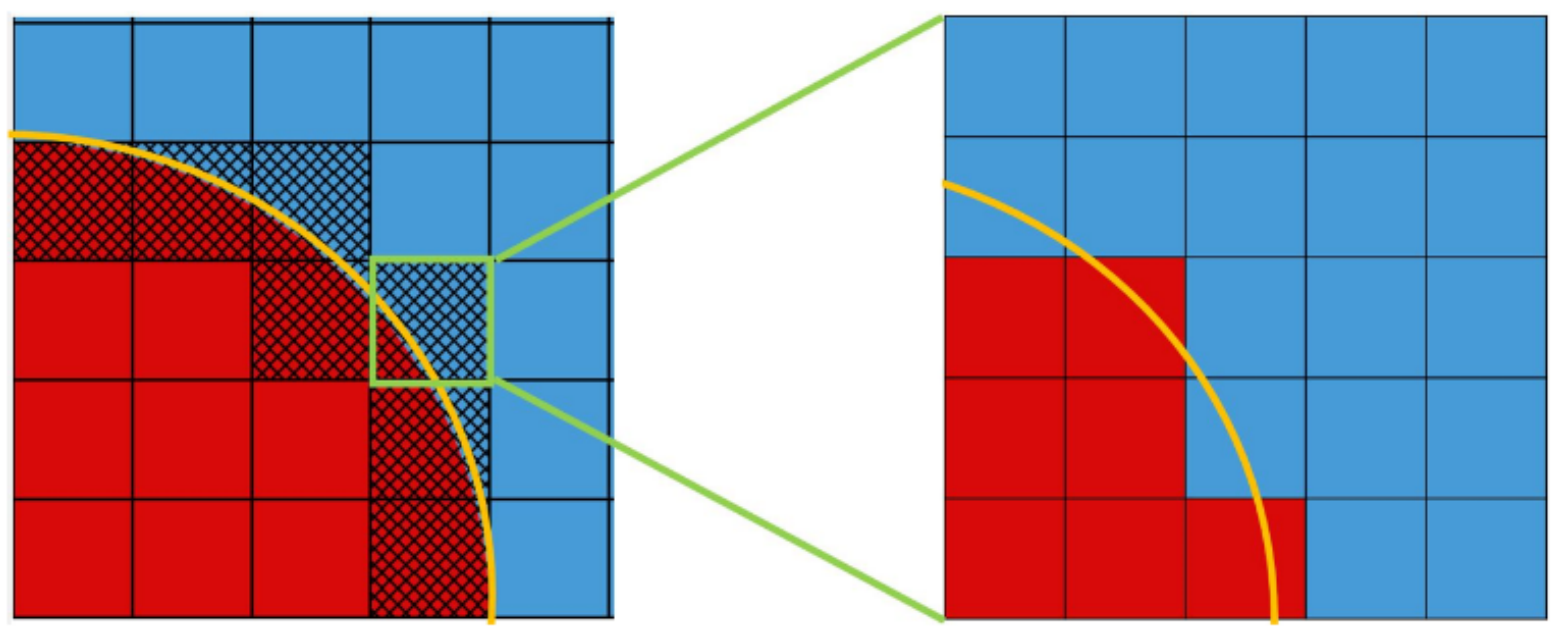

(b)

(c)

Figure 2: Voxel mesh without (a) and with (b) composite voxels; (c) describes the calculation of the volume fraction of each constituent inside the composite voxel following the method proposed by Kabel: a composite voxel consists of 25 sub-voxels, each assigned to a phase based on its centroid (the real interface is shown as an orange curve and the composites voxels are shaded in (b).) Figure (b) reprinted from [63], with permission from Elsevier

In the conventional composite voxel method, the identification of the composite voxel is determining whether a voxel contains more than one phase. In the proposed approach, instead of determining the number of phases in a voxel, a composite voxel zone is created by an artificial parameter, the composite voxel zone thickness $l$. All voxels with centers inside the zone should be considered as composite voxels. Fig. 3 (red color voxels are 
phase A and blue ones are phase B) uses a simple example to describe this new approach for two phases. The steps are as follows:

- (Fig. 3(a)) Identify the boundaries between each phase and the other phases. Parametric representations of phases boundaries can be used if they are available. Otherwise, which is more common, the zig-zag interface identification can easily be done with commercial software or algorithms. (Fig. 3 describe the zig-zag interface)

- (Fig. 3(b)) For each phase $p$ (A or $\mathrm{B}$ in the example), calculate the minimum distance $d_{p}$ from each voxel center to the phase boundary. In the signed distance field $d_{p}$, positive values are assigned inside phase $p$, and negative values outside. In the example, for $d_{A}$ the distances of voxels of phase $\mathrm{A}$ are assigned as positive values and those of phase $\mathrm{B}$ are negatives, while $d_{B}=-d_{A}$.

- (Fig. 3(c)) Choose a composite voxel zone thickness value $l$. All voxels such that $\left|d_{p}\right| \leq \frac{l}{2}$ for some phase $p$ are considered as composite voxels (marked by a cross in the figure).

The next step is to calculate the volume fractions in the composite voxels. Instead of cutting a composite voxel into sub-voxels, a regularized Heaviside function is applied here to compute the volume fraction $V_{f, p}$ of each phase $p$ within a voxel:

$$
V_{f, p}=\left\{\begin{array}{l}
0, \quad d_{p}<-\frac{l}{2} \\
\frac{1}{2}\left(1+\frac{2 d_{p}}{l}+\frac{\sin \left(\frac{2 d_{p} \pi}{l}\right)}{\pi}\right), \quad\left|d_{p}\right| \leq \frac{l}{2} \\
1, \quad d_{p}>\frac{l}{2}
\end{array}\right.
$$

Based on the properties of signed distance functions, the calculation of the normal vector is given as:

$$
\tilde{n}:=\nabla d_{P}, \quad n=\frac{\tilde{n}}{\|\tilde{n}\|}, \text { with } P=\underset{p}{\arg \max } V_{f, p}
$$

In numerical calculations, the gradient in Eq. (13) is calculated by finite differences. Finally, the homogenized properties of composite voxels are calculated using Eqs. (10) and (11). With this optimization, a parametric representation of the interface is not required, and there is no need for sub-voxels. 


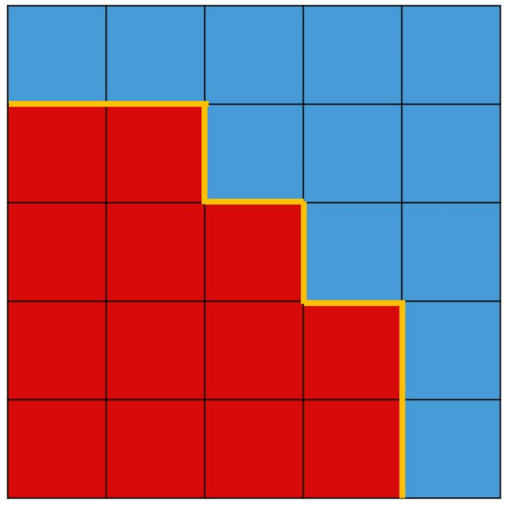

(a)

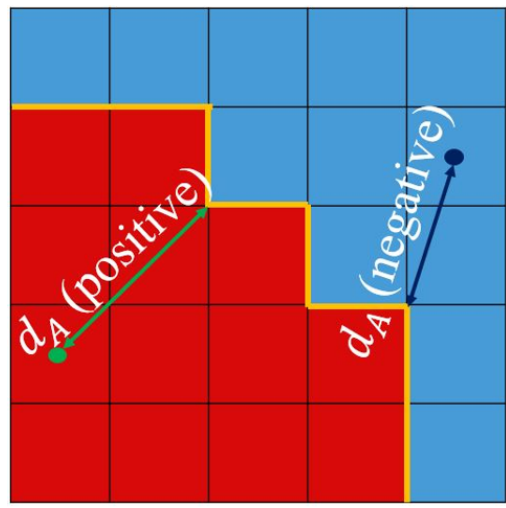

(b)

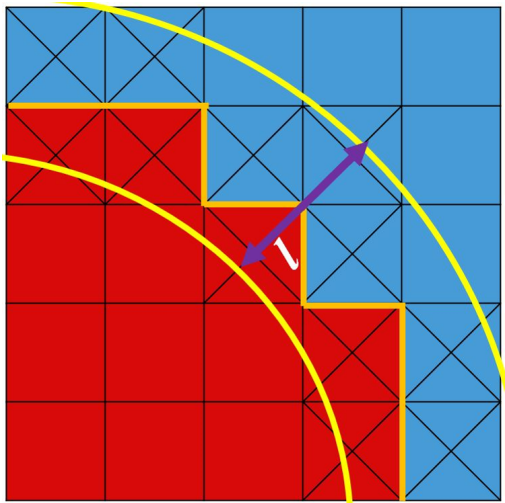

(c)

Figure 3: Illustration of the improved composite voxel method: (a) Identify the interface; (b) Calculate the minimum distance $d_{A}$; (c) Choose $l$ and determine composite voxels. The orange lines are the zig-zag interface, the green and black point in (b) are sub-voxels center and the yellow curves in (c) delimit the composite voxel zone

\subsection{Neighbour voxels average method}

Apart from the composite voxel method, which can easily be integrated within the FFT method, a post-processing technique could also be applied for reducing the oscillations, which is called the neighbour voxels average method. This kind of approach is proposed in [65] in FFT solvers and is proposed in [66] to reduce the oscillations in FEM. The oscillations in FFT and FEM can be also called the "check-board patterns" present in local strain and stress fields (as shown in Fig. 1). It can be noticed in simulation results that if the value of strain or stress in a voxel is larger than the real value, the values in the neighbouring voxels will generally be smaller. The idea is then to average these neighbour voxels results to reduce the effect of oscillations. Based on the paper [66] this method improves the accuracy of strain and stress fields as well as damage patterns when applied to a voxel mesh FEM.

In this method, it is necessary to determine the size of the averaging window around each voxel. As mentioned in [66], for different voxel mesh densities and resolutions, it is difficult to determine a constant window size. In order to define this window size, we first define the notion of layer. For a given voxel, layer $L_{0}$ is a set containing only this 
voxel. Then, layer $L_{i+1}$ is the set of voxels which are of the same phase as $L_{0}$ and whose boundaries intersect a voxel in layer $L_{i}$. This intersection can be a face, an edge or even a voxel corner. In Fig. 4(a), layer $L_{0}$ which contains only the voxel where the local averaged response is calculated is shown in orange and layer $L_{1}$ in red.

The window size is defined as the layer count $N_{L}$ such that the neighbour voxels considered for the averaging are all voxels contained in the sets $L_{i}, i \leq N_{L}$. For instance, in Fig. 4(a) the window size is $N_{L}=1$, while in Fig. 4(b) it is $N_{L}=2$. A weight function can also be introduced to reduce the influence of voxels that are inside the window but far away from its center. In general, there are three types of weight functions (mentioned in [66]) that can be applied: constant, linear, and exponential function. Based on the results in [66], these three weight functions show almost no difference in terms of oscillations reduction. Based on our recent work, we choose a linear weight function: the weight for the voxel in layer $L_{0}$ is one, and it decreases linearly with respect to the layer number $i$ down to a weight of zero for layers $L_{i}, i>N_{L}$.

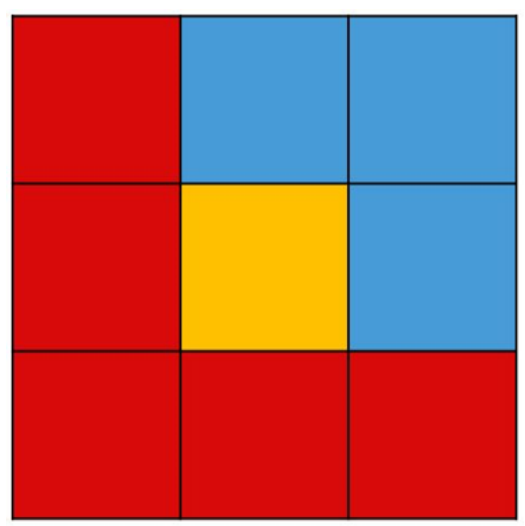

(a)

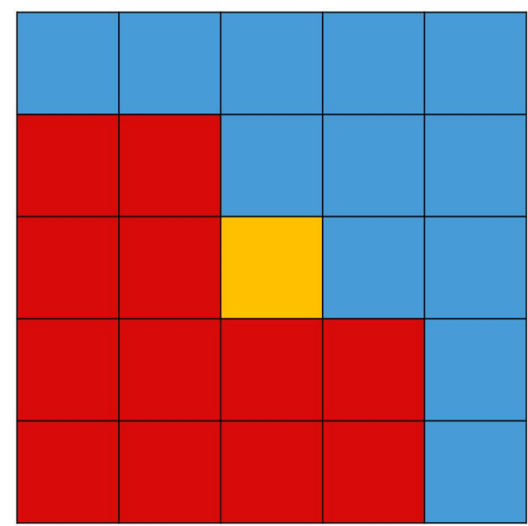

(b)

Figure 4: Layers used for averaging with different window sizes: (a) $N_{L}=1$ and (b) $N_{L}=2$. The red and blue voxels represent different phases

To summarize, the composite voxel method with our improvement and the neighbour voxels average method have been presented. Their performance in terms of oscillation reduction will be shown and discussed in Section 4.3. 


\section{Results and Discussions}

As mentioned in Section 1, this paper focuses on the numerical methods which demands that our conclusions should be as general and universal as possible. However, it is difficult to generate a universal model for complex structures like textile composites and short fibers composites. Therefore, in this paper, instead of creating one general model, three simple but representative models are designed as shown in Fig. 5. Note that these are 3D models, even though only one voxel is used in the $z$-direction. The fiber volume fraction is set to $V_{f}^{A}=0.6$ for model $\mathrm{A}, V_{f}^{B}=0.36$ for model B and $V_{f}^{C}=0.55$ for model C. For all three models, the phase A (red part) is assigned as fiber and the phase B (blue part) as matrix. Fibers are composed of E-Glass with elastic properties $E_{f}=E_{\text {glass }}=72 G P a$ and $\nu_{f}=\nu_{\text {glass }}=0.22$ while the matrix is composed of Epoxy PMR-15 with properties $E_{m}=E_{\text {epoxy }}=3.75 G P a$ and $\nu_{m}=\nu_{\text {epoxy }}=0.375$. All these material parameters are from paper [67].

In this section, the FFT method using the CGO of Moulinec-Suquet is denoted $\boldsymbol{M}$ - $\boldsymbol{S}$, the method using Willot's DGO (equivalent to Schneider's DGO with reduced integration) is denoted $\boldsymbol{W}-\boldsymbol{S}$ and the method using Schneider's fully integrated DGO is denoted $\boldsymbol{S F}$. Furthermore, the conventional composite voxel method is denoted $\boldsymbol{T} \boldsymbol{C} \boldsymbol{V}$ while the proposed improved composite voxel method is called $\boldsymbol{O C V}$. The neighbour voxels average method is represented by $\boldsymbol{A} \boldsymbol{V E}$. Regarding FEM, the voxel-based hexagonal FEM with reduced integration is denoted as $\boldsymbol{F} \boldsymbol{E} \boldsymbol{M R}$ while $\boldsymbol{F} \boldsymbol{E} \boldsymbol{M F}$ refers to fully integrated FEM.

\subsection{Mesh convergence test}

First of all, a mesh convergence test is performed on these three models for both FFT and FEM. The mesh resolution is denoted $R_{A}$ for model $\mathrm{A}, R_{B}$ for model $\mathrm{B}$ and $R_{C}$ for model C. It is defined here as the number of voxels used in the $x$ and $y$ directions. The resolutions used for model $\mathrm{A}$ are $R_{A}=5,15,45,135$ and 405. The same resolutions are used for model B. The fiber volume fraction is exactly obtained for both models for all chosen resolutions. For model C, the target fiber volume fraction $V_{f}^{C}=0.55$ can never be attained exactly due to the intrinsic nature of voxel meshes, even with very fine resolutions. Therefore, 


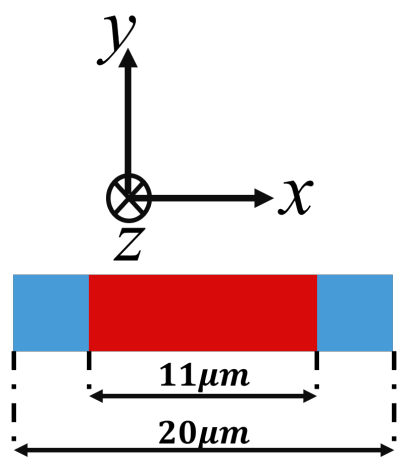

(a)

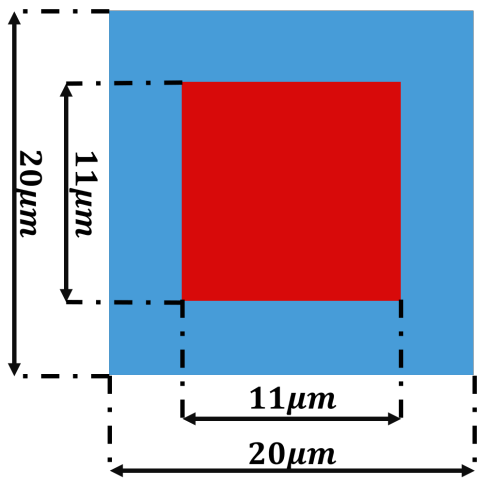

(b)

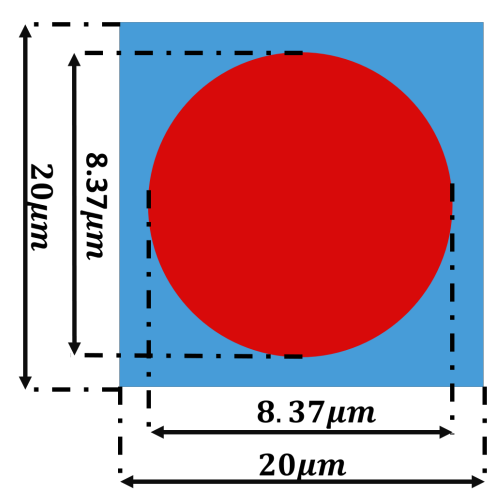

(c)

Figure 5: Illustration of the three models: (a) Model A; (b) Model B and (c) Model C

for model $\mathrm{C}$, the first step is to verify the fiber volume fraction convergence by varying the mesh resolution. Then, for all models, the convergence in terms of macroscopic properties should be verified. As mentioned in many papers, $\boldsymbol{F E \boldsymbol { R }}$ is sufficient to predict the elastic properties, thus, it is taken as the reference in this part.

As shown in Fig. 6(a) and (b), where the mesh convergence of model A is presented, the macroscopic properties for model A do not show any sensitivity to the model resolution. Different FFT methods as well as FEM show good consistency for all resolutions on model A with respect to $E_{x}$ and $G_{x y}$, where $E_{x}$ means Young's modulus in $x$-direction and $G_{x y}$ stands for shear modulus in $x y$ plane. As shown in Fig. 6(c) and (d), macroscopic properties for model $\mathrm{B}$ are computed accurately only for $R_{B}=135$ or higher. The convergence for $E_{x}$ occurs with iteratively improving lower bounds for all methods except for $\boldsymbol{S F}$ which gives higher bounds. For $G_{x y}$, all methods give higher bounds.

As mentioned above, because of the intrinsic characteristic of model $\mathrm{C}$, the mesh convergence of fiber volume fraction is first verified, as presented in Fig. 7. The mesh convergence for fiber volume fraction begins at $R_{C}=75$ (which corresponds to the red circle in Fig. 7). Based on this test, the resolutions chosen with respect to the tests of elastic properties convergence are $R_{C}=75,225,375$, which corresponds to a small, medium, and large resolution respectively. 


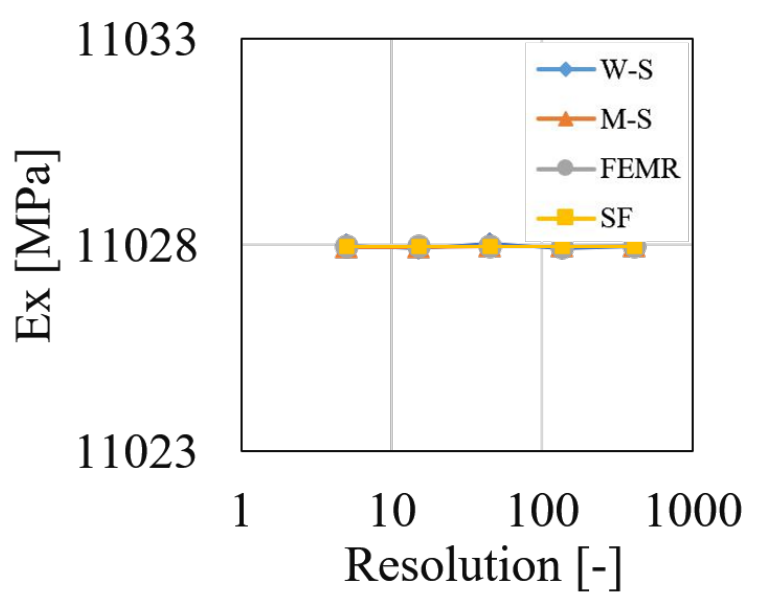

(a)

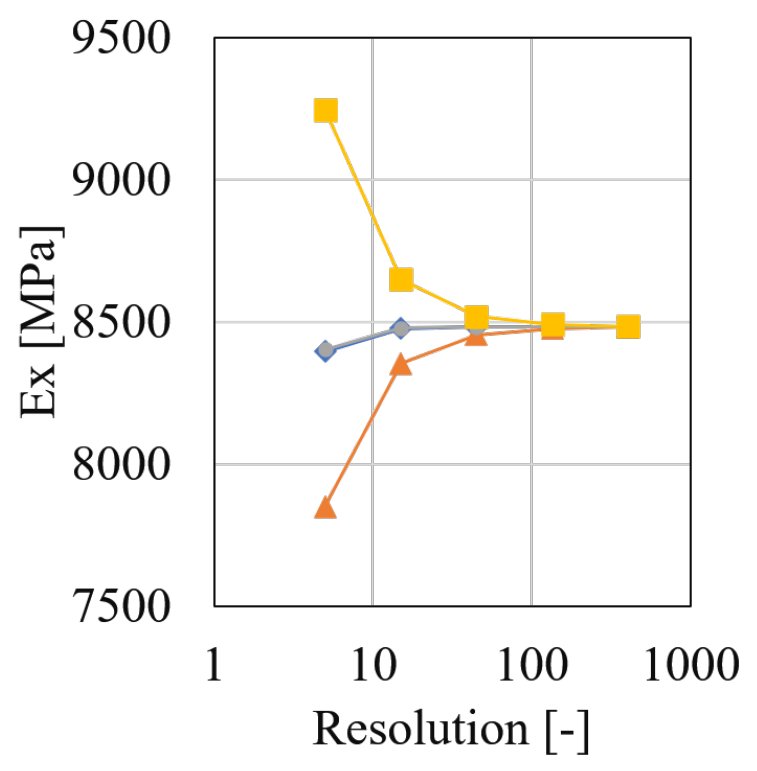

(c)

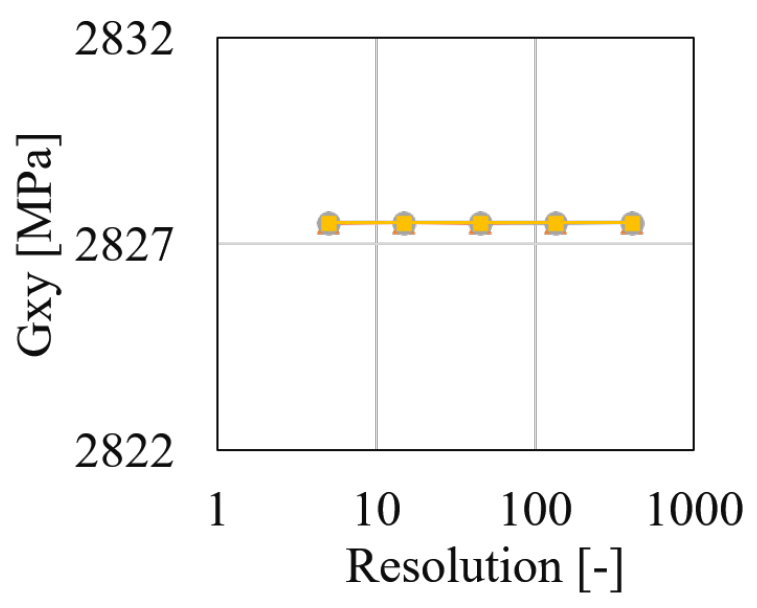

(b)

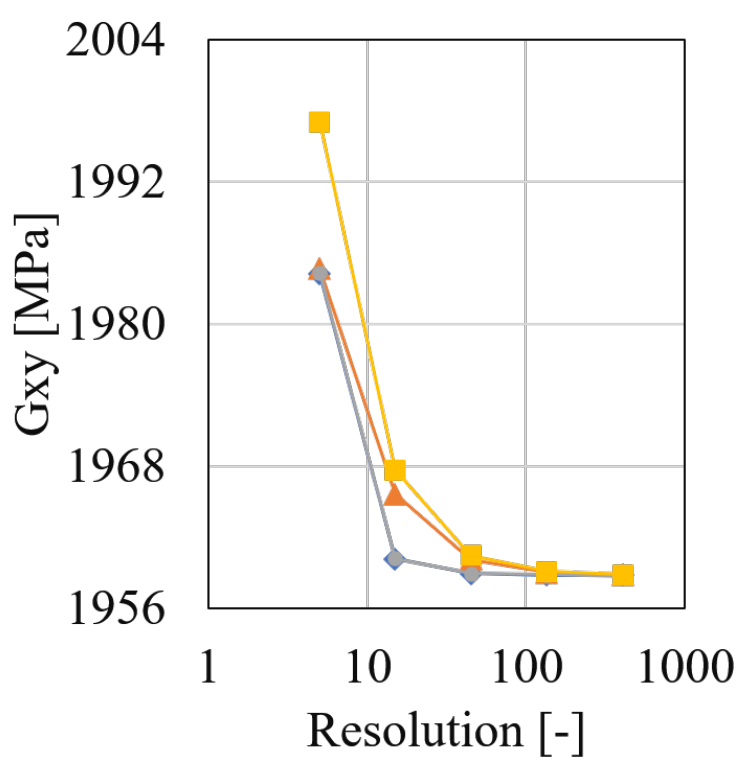

(d)

Figure 6: The variation of elastic properties $\left(E_{x}\right.$ and $\left.G_{x y}\right)$ of different FFT algorithms with different resolutions: (a) and (b) are for model A; (c) and (d) are for model B (It should be remarked that in all four figures, the curves of $\boldsymbol{W}-\boldsymbol{S}$ are cached under those of FEMR)

Table 1 presents the mesh convergence of model C. The Relative Errors of Properties $(R E P)$ are calculated as follows

$$
R E P=\frac{E_{F F T}-E_{F E M R}}{E_{F E M R}}
$$




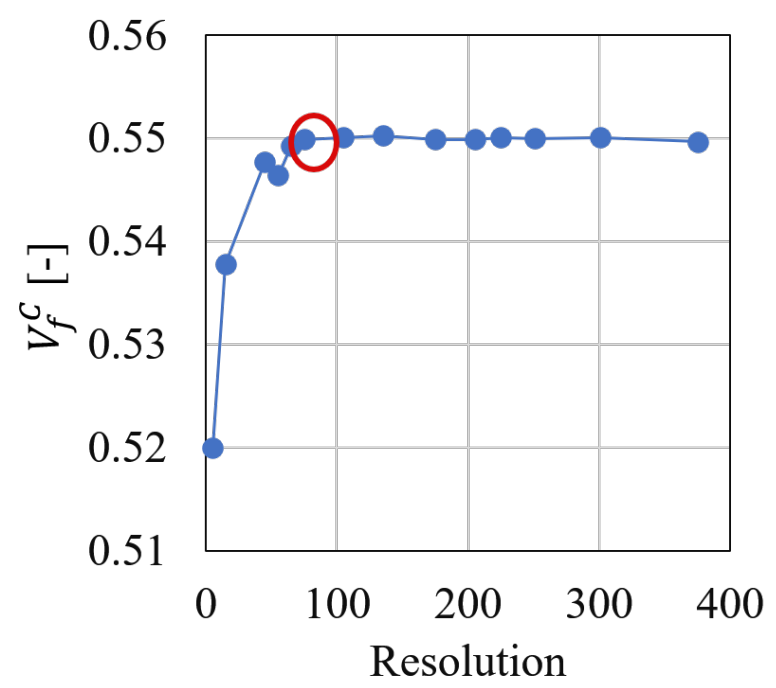

Figure 7: The variation of fiber volume fraction of model $\mathrm{C}$ with different resolution

where $E$ represents the elastic modulus $\left(E_{x}\right.$ or $\left.G_{x y}\right)$. As shown in Table 1, the relative errors of all FFT methods are smaller than $1 \%$ for $R_{C} \geq 225$. Therefore, for the next sections, the chosen resolutions for model A and model $\mathrm{B}$ are $R_{A / B}=135,405$, while for model C, the chosen resolutions are $R_{C}=225,375$.

\subsection{Causes of oscillations}

In this part, all three models are subjected to a macroscopic strain along the $x$ - direction (transverse direction) with a value $\langle\epsilon\rangle_{x x}=0.001$ while stress-free conditions are imposed in the other directions $\left(\langle\sigma\rangle_{y y}=\langle\sigma\rangle_{z z}=\langle\sigma\rangle_{x y}=\langle\sigma\rangle_{x z}=\langle\sigma\rangle_{y z}=0\right)$. These mixed-type loading conditions are applied using the method presented in [68]. It is reminded that periodic boundary conditions are intrinsic to FFT methods. Due to stress-free boundaries, Poisson's effect is active during loading.

Based on the mesh convergence test, the resolutions chosen for model A and model $\mathrm{B}$ are $R_{A / B}=135,225,315$ and 405. The resolutions chosen for model $\mathrm{C}$ are $R_{C}=225$, $255,305,345,375$ and 455 . The fiber volume fractions in this part are kept to $V_{f}^{A}=0.6$ for model A, $V_{f}^{B}=0.36$ for model B and $V_{f}^{C}=0.55$ for model C.

In order to compare the influence of oscillations, the first step is to define the crite- 


\begin{tabular}{|c|c|c|c|c|}
\hline \multicolumn{5}{|c|}{$R_{C}=75, V_{f}^{C}=0.5499$} \\
\hline & FEMR & W-S & M-S & SF \\
\hline$E_{x}(\mathrm{MPa})$ & 13373.63 & 13370.74 & 13304.28 & 13517.49 \\
\hline$R E P$ & Reference & $-0.027 \%$ & $-0.52 \%$ & $1.1 \%$ \\
\hline \hline$G_{x y}(\mathrm{MPa})$ & 2806.507 & 2806.144 & 2813.617 & 2827.081 \\
\hline$R E P$ & Reference & $-0.013 \%$ & $0.35 \%$ & $0.73 \%$ \\
\hline
\end{tabular}

\begin{tabular}{|c|c|c|c|c|}
\hline \multicolumn{5}{|c|}{$R_{C}=225, V_{f}^{C}=0.5501$} \\
\hline & FEMR & W-S & M-S & SF \\
\hline$E_{x}(\mathrm{MPa})$ & 13271.33 & 13270.66 & 13247.26 & 13318.85 \\
\hline$R E P$ & Reference & $-0.051 \%$ & $-0.18 \%$ & $0.35 \%$ \\
\hline \hline$G_{x y}(\mathrm{MPa})$ & 2792.032 & 2791.960 & 2794.563 & 2798.924 \\
\hline$R E P$ & Reference & $-0.0026 \%$ & $0.091 \%$ & $0.25 \%$ \\
\hline
\end{tabular}

\begin{tabular}{|c|c|c|c|c|}
\hline \multicolumn{5}{|c|}{$R_{C}=375, V_{f}^{C}=0.5497$} \\
\hline & FEMR & W-S & M-S & SF \\
\hline$E_{x}(\mathrm{MPa})$ & 13230.77 & 13229.38 & 13216.68 & 13258.79 \\
\hline$R E P$ & Reference & $-0.011 \%$ & -0.11 & $0.21 \%$ \\
\hline \hline$G_{x y}(\mathrm{MPa})$ & 2785.611 & 2785.473 & 2786.784 & 2789.513 \\
\hline$R E P$ & Reference & $-0.0050 \%$ & $0.042 \%$ & $0.14 \%$ \\
\hline
\end{tabular}

Table 1: The elastic properties $\left(E_{x}\right.$ and $\left.G_{x y}(\mathrm{MPa})\right)$ of model $\mathrm{C}$ of different FFT algorithms with different resolutions and their relative error compared to the FEM

rion to quantify their presence. Researchers often use the stress field to quantify the oscillations (e.g. [46]). However, even though the fiber and the matrix present the same 
amplitude of oscillations under some conditions, the oscillations in the fiber are often stronger than in the matrix because the fiber stiffness is normally higher than that of the matrix. Furthermore, when comparing different algorithms, researchers often put the color maps together and compare them visually, which is not accurate enough because two different values can have the same color on color maps. A better choice is to draw a section line and plot the strain curves of different methods. If there are some oscillations present in the curve, then the curve will not be as smooth as it should.

In order to quantify this irregularity, it is proposed to investigate the function $D_{j}$ which is defined as the increment in strain values along a curve:

$$
D_{j}=\epsilon_{j+1}-\epsilon_{j}
$$

where $\epsilon_{j}$ is the local strain at abscissa $j$ in the curve, and $\epsilon_{j+1}$ is the local strain value at the next abscissa. These can be computed for a curve of strain values along any cross line of the model. As proven in Appendix A, the local fields of $\boldsymbol{F E} \boldsymbol{M F}$ have no oscillations and can thus be considered as reference for the error measure:

$$
M D_{j}=\left|D_{j}^{F F T s / F E M R}-D_{j}^{F E M F}\right|
$$

where |.| means the absolute value. Considering that the voxel position takes the same value independently of the method, if one method has fewer oscillations, its $D_{j}$ value should be closer to that of $\boldsymbol{F E M F}$, which means that higher $M D_{j}$ values mean stronger oscillations.

Figs. 8, 9 and 10 present the average of $M D_{j}$ over each cross section line (denoted $\overline{M D}$ ) for model $\mathrm{A}, \mathrm{B}$ and $\mathrm{C}$ respectively, which can be used to evaluate the impact of each cause of oscillations. In Figs. 8 and 9, the local strain component $\epsilon_{x x}$ is chosen to study the oscillations while the radial strain component $\epsilon_{r r}$ and the tangential strain component $\epsilon_{\theta \theta}$ are chosen instead in Fig. 10.

As shown in Fig. 8, all methods show no oscillations for model A even the original $\boldsymbol{M}-\boldsymbol{S}$ algorithm, which confirms the results shown in [22]. As mentioned in the introduction, 


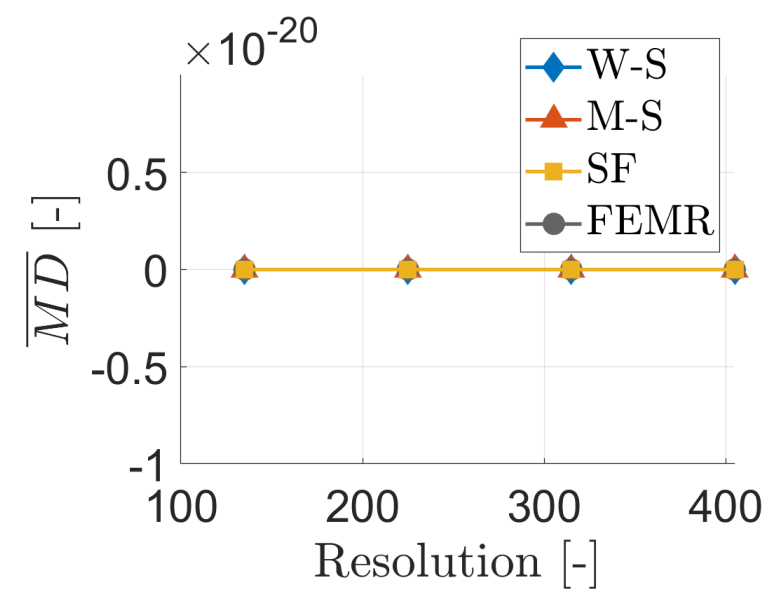

Figure 8: The value of $\overline{M D}$ for model A using the different methods

some researchers believe that one of the oscillations causes is the Gibbs phenomenon, which describes the intrinsic defect of the spectral method when dealing with high contrast signals. This does not seem applicable to composites homogenization since there is no Gibbs phenomenon in model A.

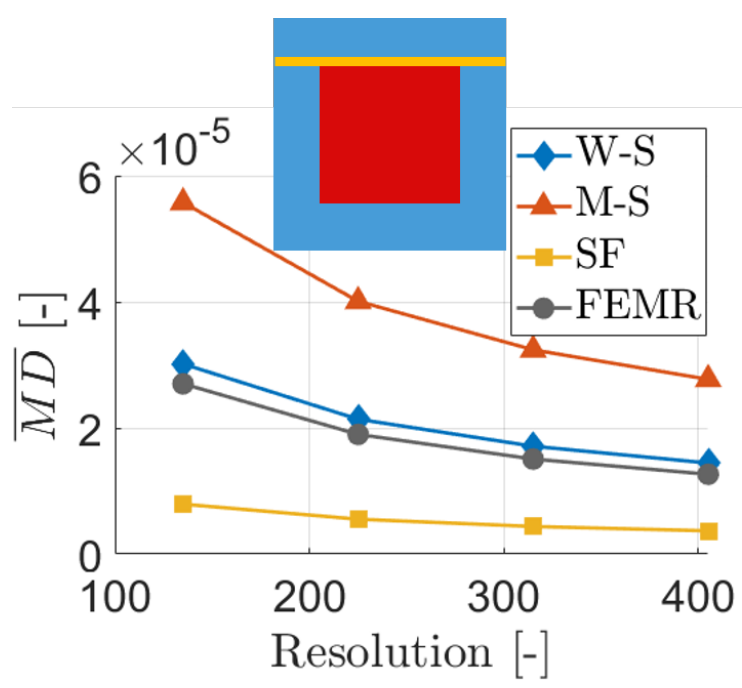

(a)

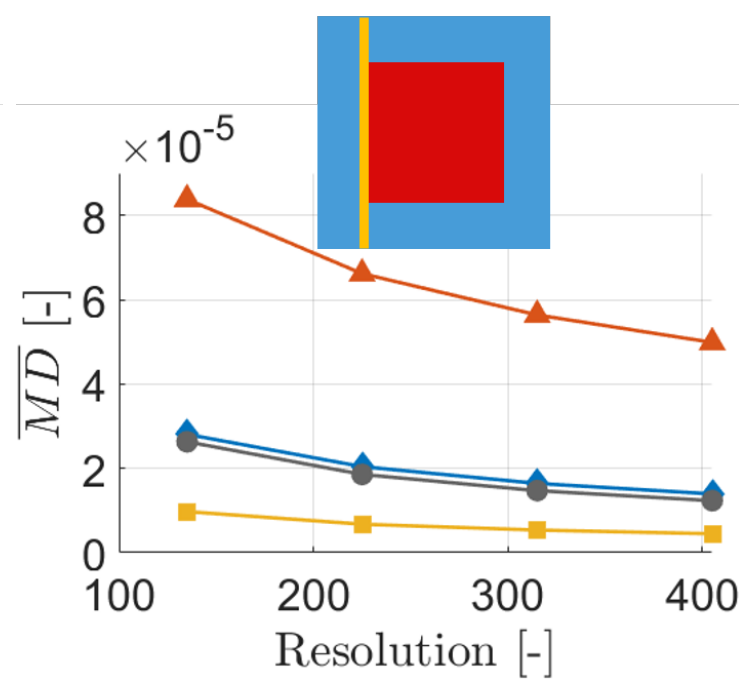

(b)

Figure 9: The values of $\overline{M D}$ for model B along (a) the horizontal interface cross line and (b) those of the vertical interface cross line

To better visualize the oscillations in FFT and in FEM, the local strain components under different approaches of model B and C are plotted in Fig. B.1 of Appendix B, where the oscillations can clearly be seen. For model B, Fig. 9(a) shows the value of 
$\overline{M D}$ along the horizontal interface cross line. Fig. 9(b) shows the $\overline{M D}$ value along the vertical interface cross section line. It should be noted that the values are taken at the matrix side which is more critical during loading (orange line in Fig. 9). Clearly, FFT methods based on a DGO reduce the oscillations significantly. Algorithm $\boldsymbol{S F}$ appears as the best choice in terms of oscillations reduction. $\boldsymbol{W}-\boldsymbol{S}$ algorithm takes the second place and the $\boldsymbol{M}-\boldsymbol{S}$ method is the worst. Furthermore, it should be paid attention that the $\boldsymbol{F} \boldsymbol{E M R}$ gives very similar results to $\boldsymbol{W}-\boldsymbol{S}$ while the oscillations are almost invisible on $\boldsymbol{F} \boldsymbol{E} \boldsymbol{M F}$, where a full integration method is applied. This phenomenon confirms that the hourglass effect is also one of the causes of oscillation in FFT methods, as already reported in [47]. This is because, like in reduced integration FEM, the strain calculated by FFT solvers is also computed at the centroid of the voxel, except for the $\boldsymbol{S F}$ method. This also explains why the latter shows less oscillations. Fig. 9 also shows that the oscillations are reduced when refining the mesh, as refining the mesh increases the sampling frequency. That proves that the loss of high frequency when discretizing the Fourier transform to a finite domain leads to oscillations, which are reduced using a DGO.

Unlike models A and B, where both FFT methods and FEM rely on voxel meshes that are body-fitted (conformal), model $\mathrm{C}$ cannot rely on a mesh that is both voxel-based and conformal. Considering that the non-smooth interface is one of the causes of oscillations, $\boldsymbol{F E} \boldsymbol{M F}$ cannot be chosen as a reference anymore. In consequence, an FEM simulation with conformal mesh with reduced integration formulation is introduced to be used as a reference, so that Eq. (14) becomes

$$
M D_{j}=\left|D_{j}^{F F T s / F E M s}-D_{j}^{\text {conformal }}\right|
$$

Due to this choice of reference result, all calculations for model $\mathrm{C}$ do not share the same mesh, even for the same resolution. Therefore, a linear interpolation is applied on the conformal mesh to get the $D_{j}$ at the same abscissa as used in voxel meshes. In Fig. 10, the $\overline{M D}$ values for model $\mathrm{C}$ are presented. Instead of using $\epsilon_{x x}$ to evaluate the oscillations, the radial strain component $\epsilon_{r r}$ and the tangential strain component $\epsilon_{\theta \theta}$ are chosen, and $D_{j}$ is computed along the interface arc on the matrix side. As shown in Fig. 10(a,b), $\boldsymbol{W}$-S and $\boldsymbol{F E M R}$ show similar behavior while $\boldsymbol{S F}$ and $\boldsymbol{F E M F}$ are also similar. It 
should be noted that the $\overline{M D}$ values shown in Fig. 10 are at least five times larger than those shown in Fig. 9 for model B, which means that the oscillations caused by nonsmooth interfaces are much more critical than other causes such as the hourglass effect. Nevertheless, the oscillations present in model $\mathrm{C}$ do not seem to decrease with increasing resolutions (sampling frequencies). We did not investigate this further. Besides, it is not possible to explain why there are less oscillations with $\boldsymbol{M}-\boldsymbol{S}$ in that regard. This is also left for future study.

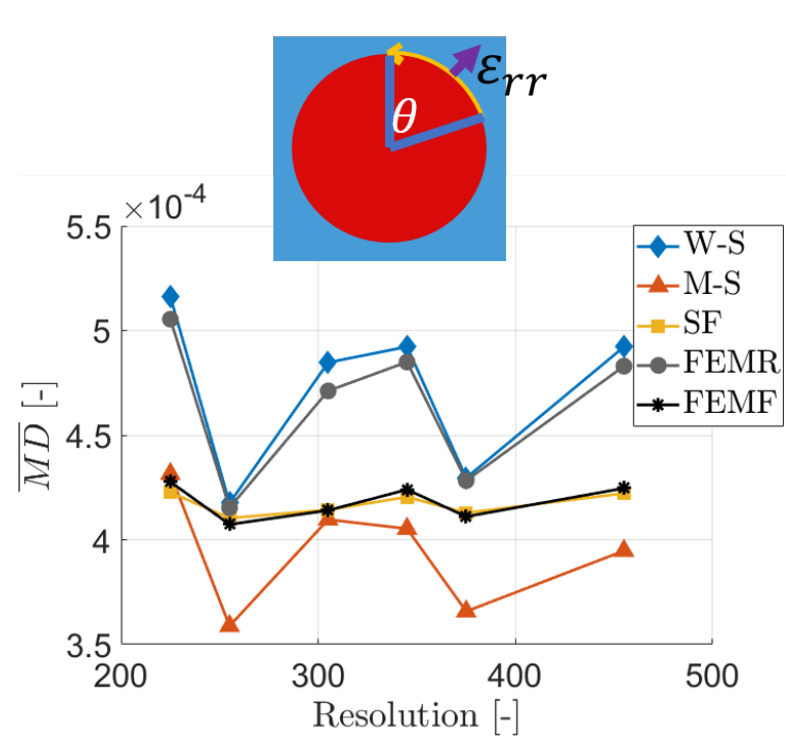

(a)

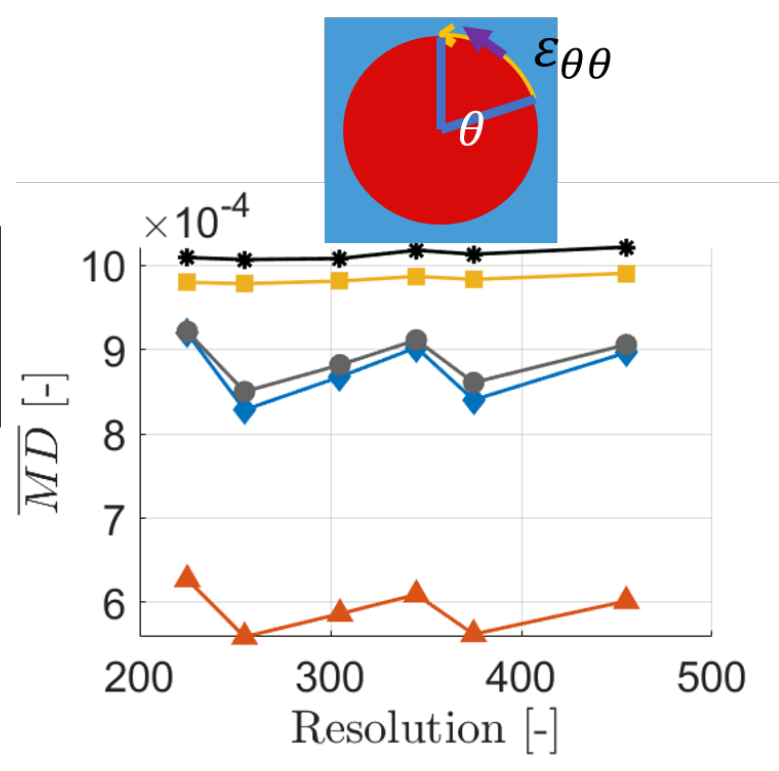

(b)

Figure 10: The $\overline{M D}$ values for model $\mathrm{C}$ (a) of the interface normal strain component and (b) of the interface tangential strain component

As a conclusion to these first tests, the non-smooth interface appears to be the most significant cause of oscillations. Note that non-smooth interfaces are more common in complex composite structures. The "truncated" Green operator could take second place due to its higher impact than the hourglass effect but its influence on oscillations can be reduced significantly by using a DGO. The hourglass effect takes the third place and could be overcome by using Schneider's DGO with full integration. Finally, there is no evidence of Gibbs phenomenon in our calculations. 


\subsection{Comparison of FFT methods}

In this part, the loading conditions are the same as in Section 4.2. Owing to the precision and reliability of FEM that have been proven over decades, FEM results with voxel meshes are chosen as reference to assess the performance of different FFT methods with regard to local fields. Due to our remarks on the hourglass effect, we consider FEM with reduced integration $(\boldsymbol{F E M R})$ and full integration $(\boldsymbol{F E M F})$. The chosen error measure is the local relative absolute error:

$$
R E=\frac{\left|\epsilon_{F F T X}-\epsilon_{F E M Y}\right|}{\left|\epsilon_{F E M Y}\right|}
$$

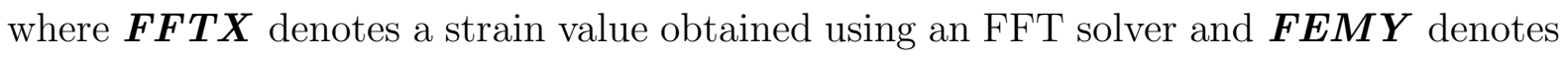
a strain value obtained using FEM with reduced or full integration. It should be noted that all strain values along the interface are taken on the matrix side. As previously, component $\epsilon_{x x}$ is the strain component chosen for comparison for models A and B while the interface normal strain $\left(\epsilon_{r r}\right)$ and the interface tangential strain $\left(\epsilon_{\theta \theta}\right)$ are chosen for model $\mathrm{C}$. We consider both the maximum of the relative absolute error on local strain values along the interface and the relative absolute error on the maximum of all local strain values along the interface. The latter is relevant for investigating the influence of oscillations on damage initiation criteria.

Results for model A are not reported herein because the relative error between FFT and FEM results, both $\boldsymbol{F E M R}$ and $\boldsymbol{F E M F}$, are null for model A. Indeed, FFT and FEM results are nearly identical for model A, independently of Green operators or reduced/full integration.

Regarding model B, Fig. 11(a,b) shows the maximum error along the interface and Fig. 11(c,d) shows the error on the maximum strain (shown in $(\mathrm{c}, \mathrm{d})$ ). In order to assess the hourglass effect, $\boldsymbol{F} \boldsymbol{E} \boldsymbol{M} \boldsymbol{R}$ is used as reference for Fig. 11(c,d) while $\boldsymbol{F} \boldsymbol{E} \boldsymbol{M F}$ is used for Fig. 11(c,d). Clearly, $\boldsymbol{W}-\boldsymbol{S}$ is the most accurate when compared with $\boldsymbol{F E M R}$ while $\boldsymbol{S F}$ is the most accurate when compared with $\boldsymbol{F E M F}$. This confirms once again that the type of integration has an influence even for FFT methods. This is verified for both error measures. Fig. 12 shows the average interface relative (orange contour) 


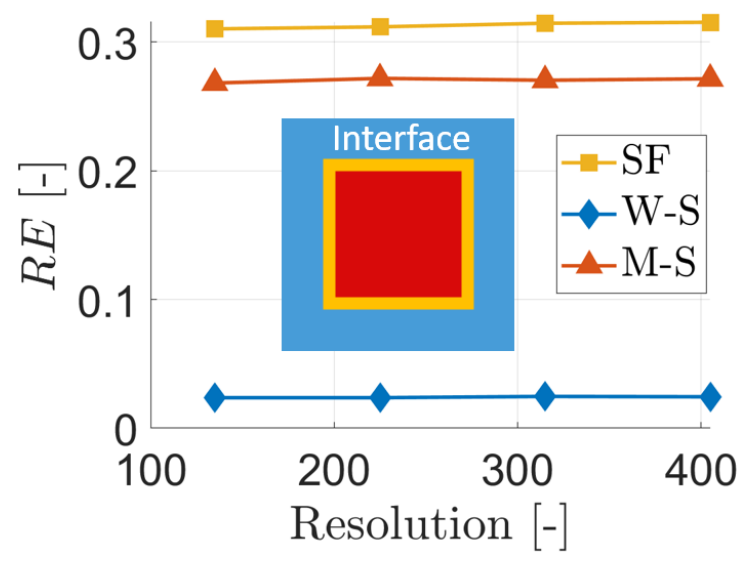

(a)

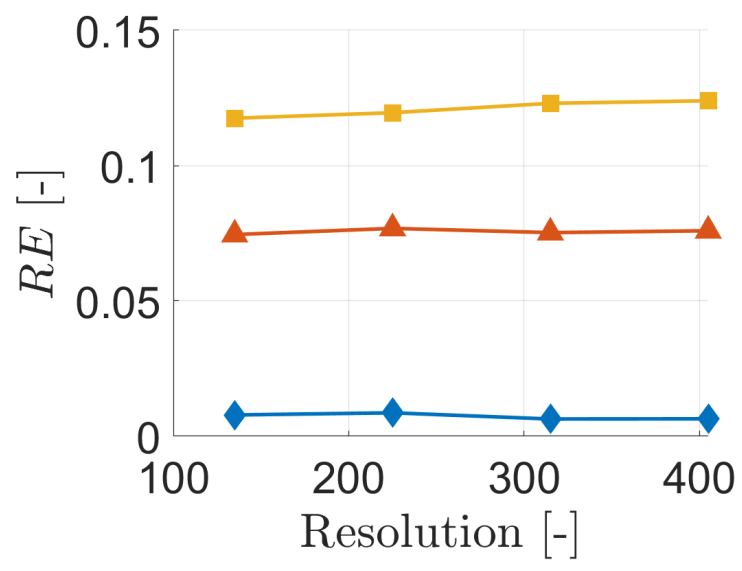

(c)

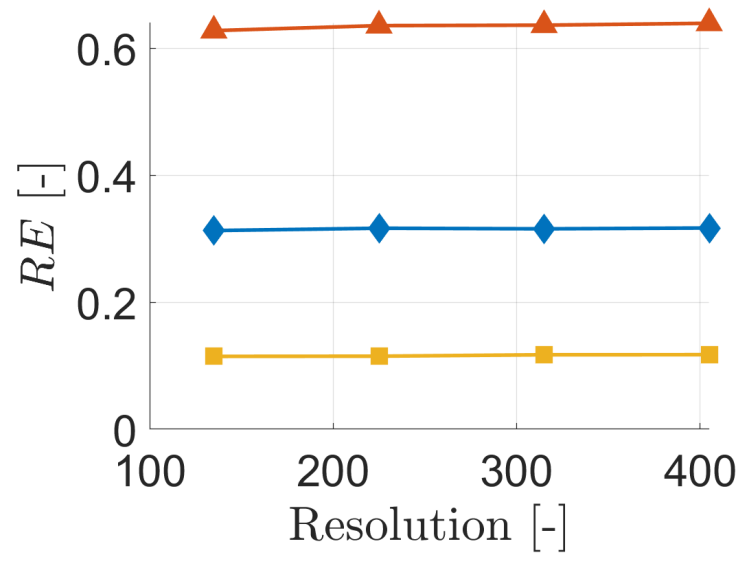

(b)

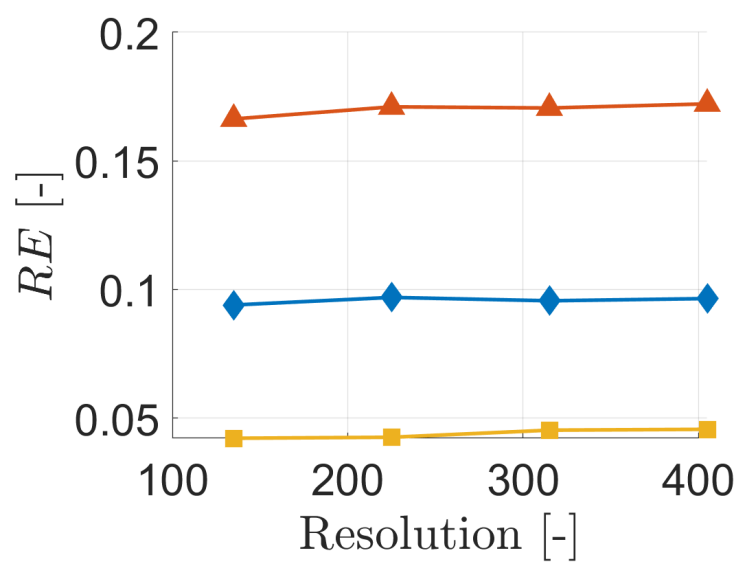

(d)

Figure 11: Maximum relative absolute error along the interface for model B: (a) with $\boldsymbol{F E M R}$ and (b) with $\boldsymbol{F E M F}$; Relative absolute error on the maximum strain along the interface (c) with $\boldsymbol{F} \boldsymbol{E} \boldsymbol{M} \boldsymbol{R}$ and (d) with $\boldsymbol{F} \boldsymbol{E} \boldsymbol{M F}$

errors $(a, b)$, the average relative errors at the fiber center (green point) $(c, d)$ and those of whole volume (e,f). Same as Fig. 11, the left parts of Fig. 12 are the relative errors with $\boldsymbol{F E} \boldsymbol{M R}$ and the right parts are those with $\boldsymbol{F E M F}$. As shown in these two figures, when compared with $\boldsymbol{F E M R}, \boldsymbol{W}-\boldsymbol{S}$ is always more accurate while $\boldsymbol{S F}$ is more accurate when compared with $\boldsymbol{F E M F}$, except for the average relative errors at the fiber center. We noticed that the $\boldsymbol{W}-\boldsymbol{S}$ method produces more oscillations at the fiber center and in general it is comparable (or equivalent) to $\boldsymbol{F} \boldsymbol{E M R}$ close to interfaces but has more oscillations in the bulk (also shown in Appendix C), which can be neglected due to their sufficient low values. 
The relative errors of model $\mathrm{C}$ compared with FEM results at different positions are presented in Appendix D. In general the conclusions are the same as for model B. $\boldsymbol{S F}$ has the best results when compared with $\boldsymbol{F E} \boldsymbol{M F}$ and it is $\boldsymbol{W}-\boldsymbol{S}$ when compared with $\boldsymbol{F E M R}$. Furthermore, the average relative errors in the volume in Fig. 12 and Appendix D both show that they will decrease when refining the mesh while the maximum errors and the errors at the maximum strain (Fig. 11 and Appendix D) show the opposite behavior. This phenomenon could be due to the increased error at singularities when refining the mesh. Because both model B and model C have sharp corners, strain concentration appears during the loading. Note that this is a physical phenomenon for model B but it is artificially caused by the ziz-zag interface for model C. Model C has many more sharp corners than model B, which could explain the opposite behavior with mesh refinement on interface average errors in model B and $\mathrm{C}$.

As a conclusion of this part, the three FFT methods give nearly exact results on model A. However, on models B and C, the $\boldsymbol{S F}$ is the most accurate when compared to the full integration FEM method and the $\boldsymbol{W}-\boldsymbol{S}$ is more accurate when compared to the reduced integration formulation. This difference between reduced and full integration was also observed in [47]. Considering that $\boldsymbol{S F}$ has fewer oscillations than others, in consequence, if a simulation needs high precision where a full integration is required in FEM, the $\boldsymbol{S F}$ should be applied when the simulation is performed using an FFT solver.

However, applying $\boldsymbol{S F}$ requires 8 times more memory than $\boldsymbol{W}-\boldsymbol{S}$ and the computation time is also much longer. In many cases, the full integration formulation is not needed and the hourglass effect can be easily controlled by introducing "hourglass stiffness" as done in most FEM codes. Furthermore, the non-smooth interface has been proven to be the most critical cause of oscillations. Thus, a reduced integration formulation could be a better choice in many cases, where the $\boldsymbol{W}-\boldsymbol{S}$ is in general more accurate than other FFT methods. In the next part, the $\boldsymbol{W}-\boldsymbol{S}$ algorithm is chosen to apply composite voxel and neighbour voxels average methods reduce the oscillations at the interface. 


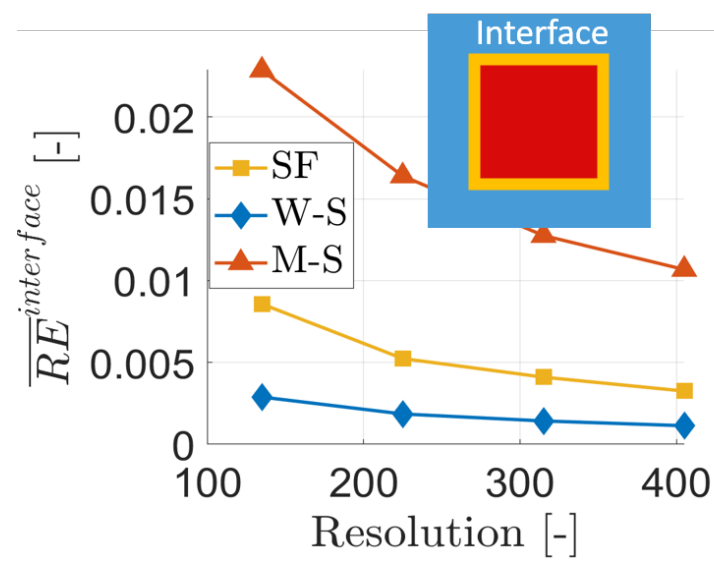

(a)

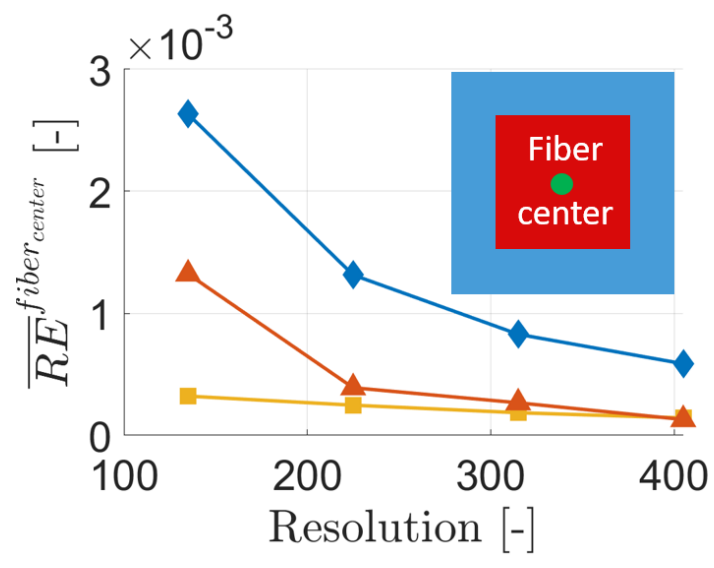

(c)

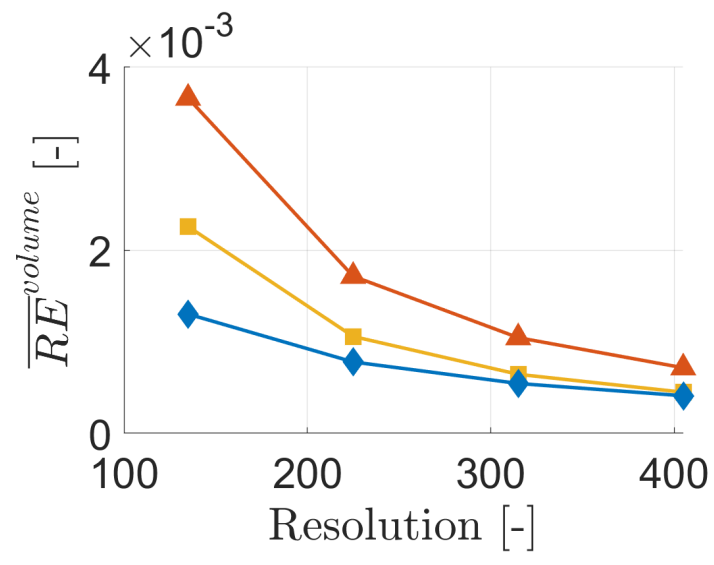

(e)

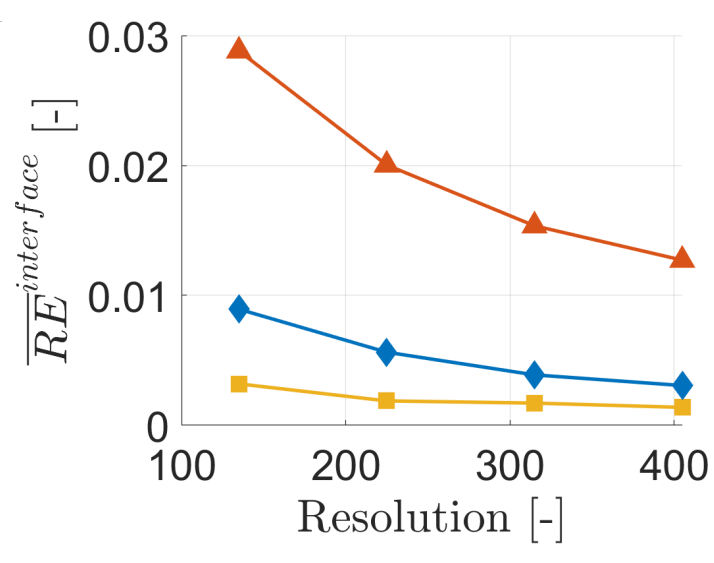

(b)

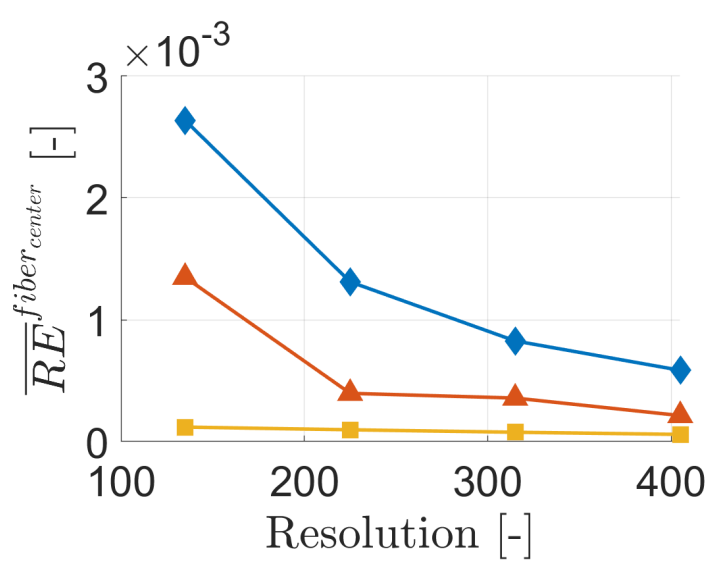

(d)

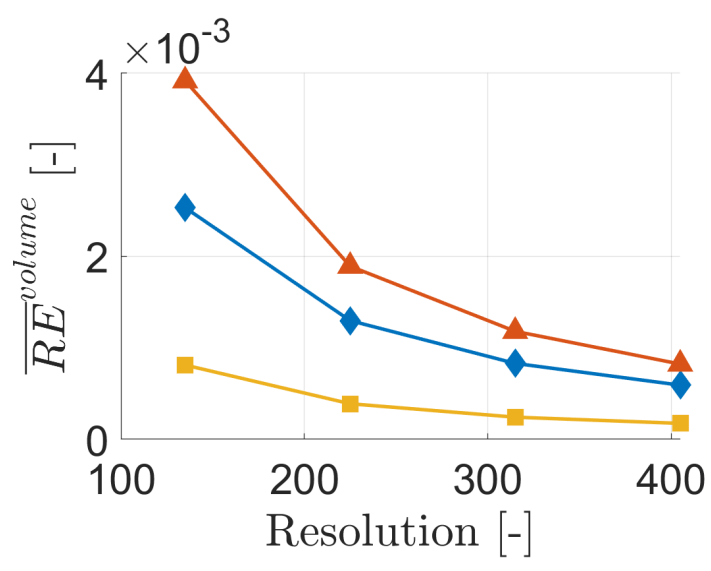

(f)

Figure 12: The average errors for FFT results along the interface compared to: (a) $\boldsymbol{F E M R}$ and (b) $\boldsymbol{F} \boldsymbol{E} \boldsymbol{M F}$; The average errors for FFT results at the fiber center compared to: (c) $\boldsymbol{F} \boldsymbol{E} \boldsymbol{M R}$ and (d) $\boldsymbol{F} \boldsymbol{E} \boldsymbol{M F}$; The average errors of FFT results over the whole volume compared to: (e) $\boldsymbol{F E} \boldsymbol{M R}$ and (f) $\boldsymbol{F} \boldsymbol{E} \boldsymbol{M F}$ 


\subsection{Comparison of interface smoothing techniques}

As proven in the previous part, the most critical cause of oscillations is the non-smooth interface, because not only it can lead the oscillations five times stronger than other causes, but also it is more common in composite materials. Therefore, the DGO-based FFT solvers are not sufficient and the composite voxel and the neighbour voxel average methods are proposed. The composite voxel method replaces the mono-material properties of a voxel by the homogenized material properties which are based on the local volume fractions of phases inside the voxel. The neighbour voxel average method averages the value of a voxel with neighbour voxels around it.

Regarding the composite voxel method, two alternatives are assessed in this paper: the conventional method which is denoted as $\boldsymbol{T} \boldsymbol{C} \boldsymbol{V}$ and the proposed improved method which is denoted $\boldsymbol{O} \boldsymbol{C} \boldsymbol{V}$. It should be noted that this composite voxel method is not related to the notion of inter-phase, which is not discussed or modeled herein.

For the improved method $\boldsymbol{O} \boldsymbol{C} \boldsymbol{V}$, it is necessary to define the composite voxel thickness zone $l$. To be consistent with the conventional method $\boldsymbol{T} \boldsymbol{C} \boldsymbol{V}, l$ should be as small as possible. However, if $l$ is chosen to be too small, some voxels at the interface have the risk to be excluded from the composite voxel zone. Thus, we chose to set $l$ as twice the voxel size, which ensures that there is always at least one layer of composite voxel at the interface, and at most two layers (one on the matrix side and the other on the fiber side).

As for the $\boldsymbol{A} \boldsymbol{V} \boldsymbol{E}$ technique, the most suitable average layer counts presented in [66] is $N_{L}=2$, thus, $N_{L}=1$ and 2 are both studied in the following. In this part, all boundary conditions are the same as in Sections 4.2 and 4.3.

Because the first layer of voxels along the interface are occupied by the composite voxels, in the next figures (Figs. 13, 14, 15, 16 and 17), the layer of voxels out of the composite voxels zone is chosen to compare the oscillations reduction by applying different techniques. Since the composite voxels zone is very small compared to the model size, the 
layer of voxels chosen from Figs. 13 to 17 can also be considered as the interface response, which can be denoted as interface ${ }^{2 n d}$ to distinguish from the real interface as shown in Sections 4.2 and 4.3 .

As shown in Fig. 13, where the interface ${ }^{2 n d}$ normal strain $\left(\epsilon_{r r}\right)$ and tangential strain $\left(\epsilon_{\theta \theta}\right)$ are drawn, the composite voxel method can reduce significantly the oscillations in the presence of a non-smooth interface. Fig. 14 clearly shows the effect of the reduction of oscillations based on the $\boldsymbol{O} \boldsymbol{C} \boldsymbol{V}$ and the $\boldsymbol{T} \boldsymbol{C} \boldsymbol{V}$ methods. For the $\boldsymbol{T} \boldsymbol{C} \boldsymbol{V}$ method, we tried using different numbers of sub-voxels in each direction. The $\boldsymbol{T} \boldsymbol{C} \boldsymbol{V}$ curves shown in Fig. 14 could be obtained using at least 41 sub-voxels in each direction. We did not see any improvement by using more sub-voxels. With our un-optimized Python implementations, the total computation time for the $\boldsymbol{T} \boldsymbol{C} \boldsymbol{V}$ method using 41 sub-voxels in each direction was of the same order as the one for the $\boldsymbol{O} \boldsymbol{C V}$ method. This is interesting as $\boldsymbol{O} \boldsymbol{C V}$ reduces the oscillations more effectively than $\boldsymbol{T} \boldsymbol{C} \boldsymbol{V}$ for both strain components and all resolutions, and is also more general because it can deal easily with non-parametrized interfaces.

To compare $\boldsymbol{O} \boldsymbol{C} \boldsymbol{V}$ and $\boldsymbol{A} \boldsymbol{V E}$, the $\overline{M D}$ values are shown in Fig. 15(a,b). These two graphs show that $\boldsymbol{O} \boldsymbol{C} \boldsymbol{V}$ reduces the oscillations more effectively than the $\boldsymbol{A} \boldsymbol{V E}$ if one layer is used but with two layers $\boldsymbol{A} \boldsymbol{V} \boldsymbol{E}$ becomes more effective. However, as shown in Fig. 16, the $\boldsymbol{A} \boldsymbol{V} \boldsymbol{E}$ cannot treat well the abnormal phenomenon on the normal interface ${ }^{2 n d}$ strain at around the 45 degree, while the $\boldsymbol{O} \boldsymbol{C} \boldsymbol{V}$ does not have this kind of issue. Besides, $\boldsymbol{A} \boldsymbol{V} \boldsymbol{E}$ is a kind of post-treatment, where the equilibrium equation and the compatibility condition are not verified. Maybe in some cases, a very high reduction of oscillations is required, where a combination of these two methods could be applied. This possibility would not be discussed in this paper. In most applications, the $\boldsymbol{O} \boldsymbol{C} \boldsymbol{V}$ seems to be a more optimal choice.

To confirm the conclusions raised when comparing interface smoothing techniques for model $\mathrm{C}$, it is necessary to verify the influence of the $\boldsymbol{O} \boldsymbol{C} \boldsymbol{V}$ technique for the two other models. For model A, results show that the relative errors remain equal to zero even 


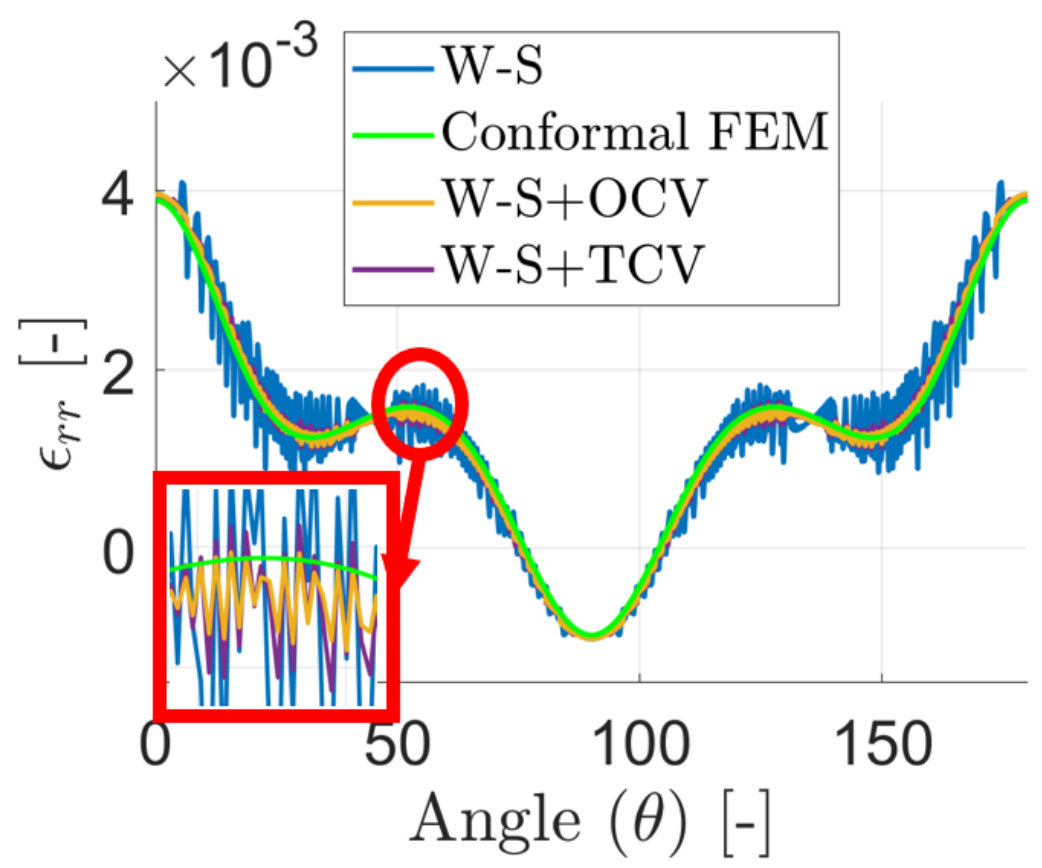

(a)

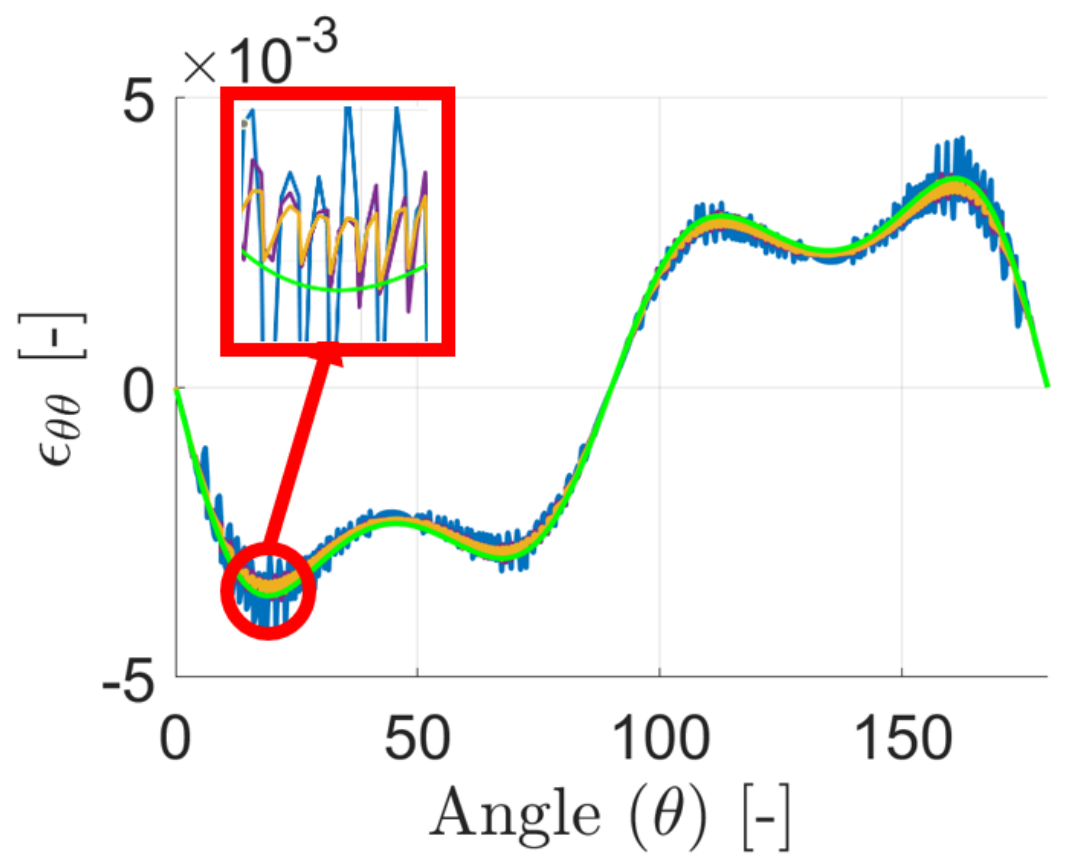

(b)

Figure 13: The interface ${ }^{2 n d}$ normal strain (a) and tangential strain (b) with $R_{C}=375$ and using FEM conformal mesh (reference result) and $\boldsymbol{W}-\boldsymbol{S}$ alone or combined with the conventional and enhanced composite voxel methods (Remark: All the strain components are taken on the matrix side with illustration figure shown in Fig. 10, and the figure should be viewed in color) 


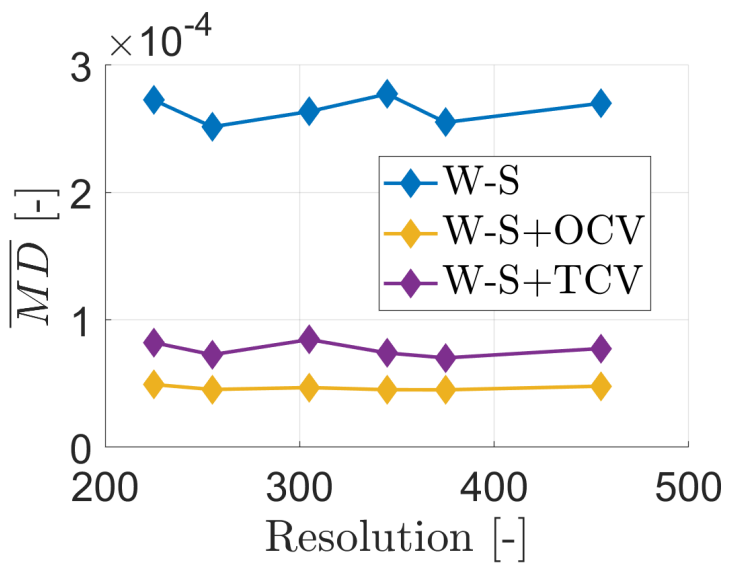

(a)

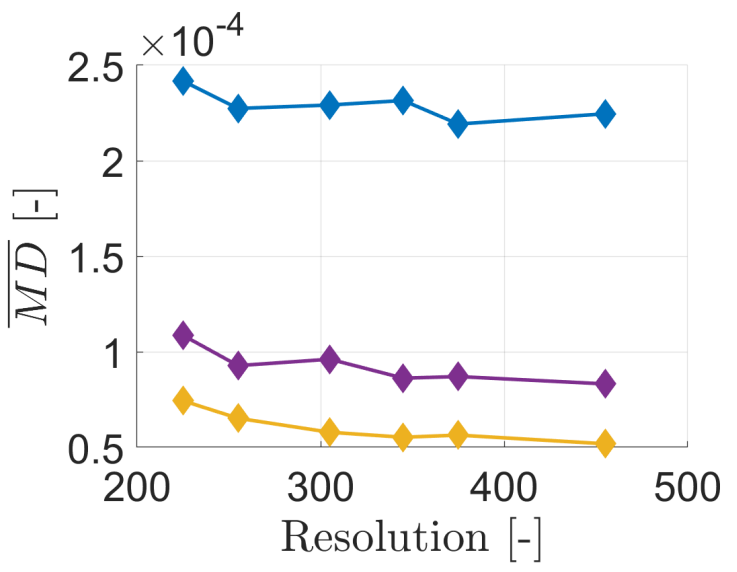

(b)

Figure 14: The $\overline{M D}$ value of interface ${ }^{2 n d}$ normal (a) and tangential (b) strains using $\boldsymbol{W}-\boldsymbol{S}$ and different composite voxels approaches. (Remark: The figure should be viewed in color)

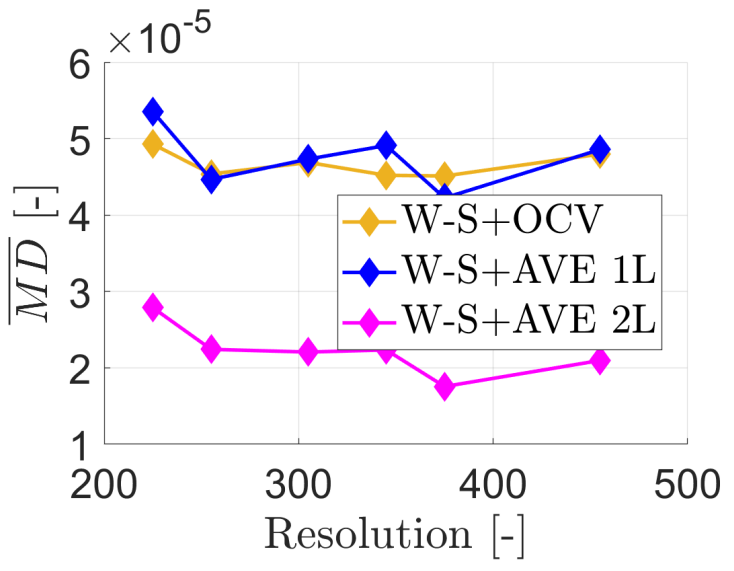

(a)

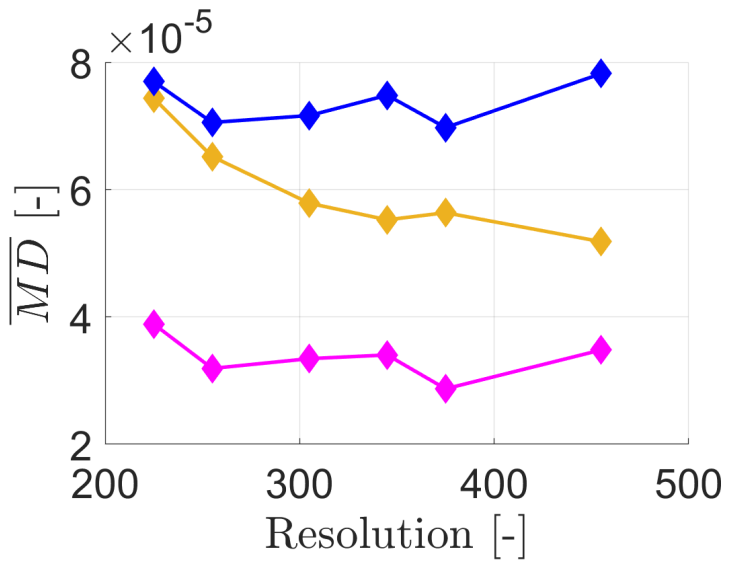

(b)

Figure 15: The $\overline{M D}$ values of interface ${ }^{2 n d}$ normal strain component (a) and of the tangential strain component (c). (Remark: AVE $1 L$ means $L_{\max }=1$ and so on. The figure should be viewed in color)

when applying the $\boldsymbol{O} \boldsymbol{C} \boldsymbol{V}$ technique except for the voxels inside the composite voxel zone. It is not possible to report relevant error measures for this zone because the fiber volume fraction is not the same between $\boldsymbol{W}-\boldsymbol{S}+\boldsymbol{O C} \boldsymbol{V}$ and $\boldsymbol{F E M R}$ at the same position.

For model B, the relative errors between $\boldsymbol{W}-\boldsymbol{S}+\boldsymbol{O C} \boldsymbol{V}$ and $\boldsymbol{F E M R}$ at interface ${ }^{2 n d}$ layer 


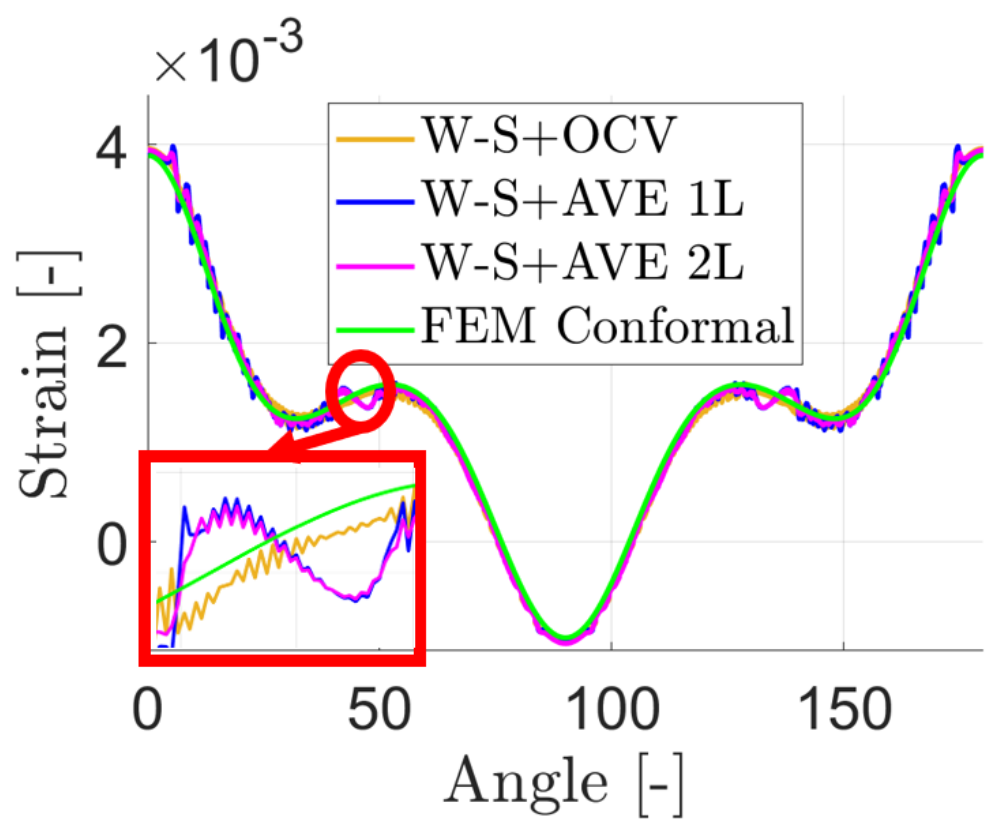

Figure 16: The interface ${ }^{2 n d}$ normal strain $\left(\epsilon_{r r}\right)$ component of different approaches of model C with $R_{C}=375$ (Remark: $A V E 1 L$ means $L_{\max }=1$ and so on. The figure should be viewed in color)

are shown in Fig. 17. The errors for $\boldsymbol{M}-\boldsymbol{S}$ and $\boldsymbol{W}-\boldsymbol{S}+\boldsymbol{A} \boldsymbol{V E} \boldsymbol{2} \boldsymbol{L}$ are also reported. Clearly, $\boldsymbol{A} \boldsymbol{V E} \mathscr{E} \boldsymbol{L}$ is not relevant for these models as it increases the errors significantly. This technique is hence only relevant for non-smooth interfaces and thus does not apply to general situations. The $\boldsymbol{O} \boldsymbol{C}$ technique also increases a little the relative error, which is quite normal because adding composite voxels artificially reduces the contrast between fiber and matrix. Nevertheless, the relative error of the $\boldsymbol{O} \boldsymbol{C} \boldsymbol{V}$ method is much lower than the $\boldsymbol{A} \boldsymbol{V E} \boldsymbol{E} \boldsymbol{L}$ method, furthermore, they are very close to the $\boldsymbol{M}-\boldsymbol{S}$ scheme. As a conclusion, the increase of the error for models $\mathrm{A}$ and $\mathrm{B}$ is acceptable for the improved composite voxel method given its simplicity in dealing with ziz-zag interfaces.

\section{Conclusion}

In this paper, Fast Fourier Transform (FFT) methods for composites homogenization have been assessed and spurious oscillations have been observed in the results they produce. The causes of these oscillations have been investigated and potential solutions to deal with these issues have been critically compared. 


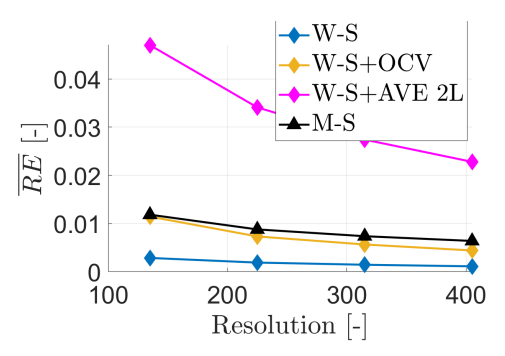

(a)

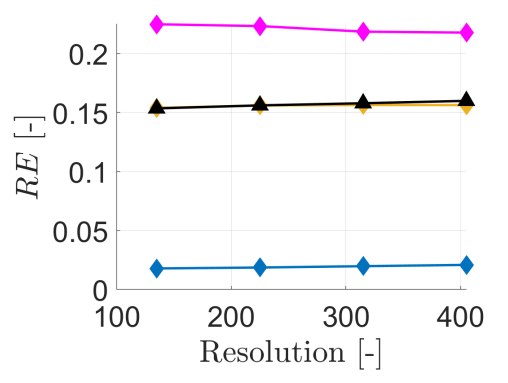

(b)

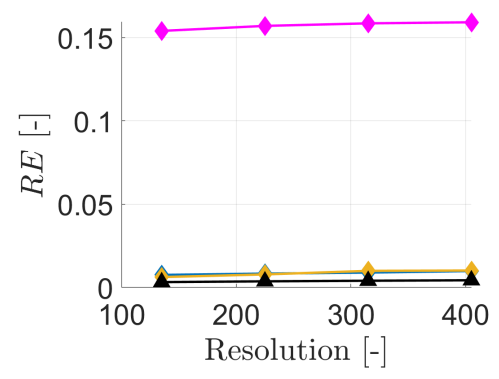

(c)

Figure 17: The relative errors of $\boldsymbol{W}-\boldsymbol{S}$ and different treating techniques with $\boldsymbol{F E M R}$ along with the layer of interface ${ }^{2 n d}$ : a). The average relative error of the layer; b). Maximum error on the layer; c). The errors of the maximum strain on the layer. (Remark: The figure should be viewed in color.)

The most severe cause of oscillation in the presence of non-smooth interfaces has been identified as the presence of the zig-zag pattern at the interface. It has been shown that it leads to oscillations five times stronger in magnitude than other causes. The second severe cause of oscillations has been identified as a defect in the original FFT method of Moulinec and Suquet $\boldsymbol{M}-\boldsymbol{S}[20,21,22]$ due to the use of a Continuous Green Operator (CGO). A solution to deal with this defect is to use a Discrete Green Operator (DGO), as in the $\boldsymbol{W}-\boldsymbol{S}$ method proposed by Willot in [60] and in the $\boldsymbol{S F}$ method proposed by Schneider in [62]. The third cause of oscillations is the hourglass effect. As in the reduced integration FEM, most FFT methods, such as the original approach $\boldsymbol{M}-\boldsymbol{S}$ and the DGO approach $\boldsymbol{W}-\boldsymbol{S}$, compute the local strain and stress only at the centroid of the voxels. This leads to oscillations under complex loading. This kind of defect can be reduced by introducing a fully integrated formulation such as $\boldsymbol{S F}$. Finally, the Gibbs phenomenon, which is believed as one of the causes of oscillations, does not seem to have an impact on oscillations based on our tests.

In terms of relative errors between FFT and FEM results, in general, $\boldsymbol{S F}$ is the most accurate when compared to a fully integrated FEM while $\boldsymbol{W}-\boldsymbol{S}$ is the most accurate when compared to a reduced integration formulation. In both cases, $\boldsymbol{M}-\boldsymbol{S}$ is the least 
accurate. In other words, both the $\boldsymbol{W}-\boldsymbol{S}$ and $\boldsymbol{S} \boldsymbol{F}$ methods deal well with the first cause of oscillations and the $\boldsymbol{S F}$ additionally deals with the hourglass effect. However, $\boldsymbol{S F}$ requires 8 times more computational resources both in terms of memory and computation time than $\boldsymbol{S F}$. Therefore, even though $\boldsymbol{S F}$ has some additional advantages in terms of oscillations reduction, $\boldsymbol{W}-\boldsymbol{S}$ seems to be the optimal choice.

The $\boldsymbol{W}-\boldsymbol{S}$ approach can be combined with the composite voxel or the neighbour voxels average methods in order to reduce the oscillations further in the case of non-smooth interfaces with zig-zag patterns due to the voxel-based discretization. This can be done with the improved composite voxel method $(\boldsymbol{O C} \boldsymbol{V})$ that is proposed in this paper. Compared with the conventional composite voxel method $\boldsymbol{T C V}$, the $\boldsymbol{O} \boldsymbol{C}$ does not require parametric representations of interfaces and can hence be applied directly to digital images. When comparing the $\boldsymbol{O} \boldsymbol{C} \boldsymbol{V}$ with the neighbour voxels average method $\boldsymbol{A} \boldsymbol{V} \boldsymbol{E}$, it is observed that $\boldsymbol{O} \boldsymbol{C} \boldsymbol{V}$ reduces the oscillations more effectively than $\boldsymbol{A} \boldsymbol{V} \boldsymbol{E}$ with one layer but less effectively than $\boldsymbol{A} \boldsymbol{V} \boldsymbol{E}$ with two layers. In addition, we noticed an increase of the error when there are no zig-zag patterns for all these smoothing techniques. This increase is more significant when using $\boldsymbol{A} \boldsymbol{V} \boldsymbol{E}$ with two layers compared to $\boldsymbol{O} \boldsymbol{C} \boldsymbol{V}$.

As a conclusion, a combination of Willot's rotated scheme $(\boldsymbol{W}-\boldsymbol{S})$ and our improved composite voxel technique seems to be the optimal choice for FFT-based composites homogenization. The damage analysis based on this method could be studied in the future.

\section{A Appendix A}

As mentioned in Section 4.2, the output values from numerical calculation are not differentiable. However, the finite difference could be used to calculate the smoothness of a curve. Thus, the Finite Difference of First Order (FDFO) between two voxels are calculated to evaluate the smoothness of the method $\boldsymbol{F E M F}$.

In general, a calculation of FDFO between two voxels means calculating the slope the 


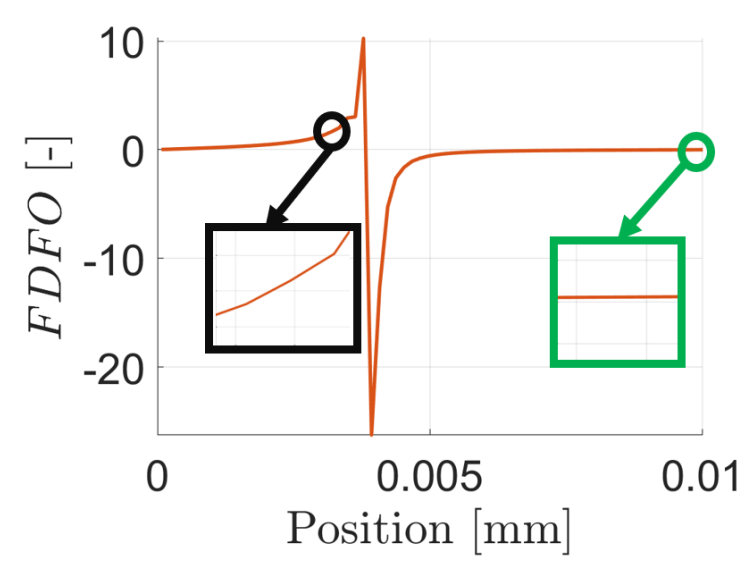

(a)

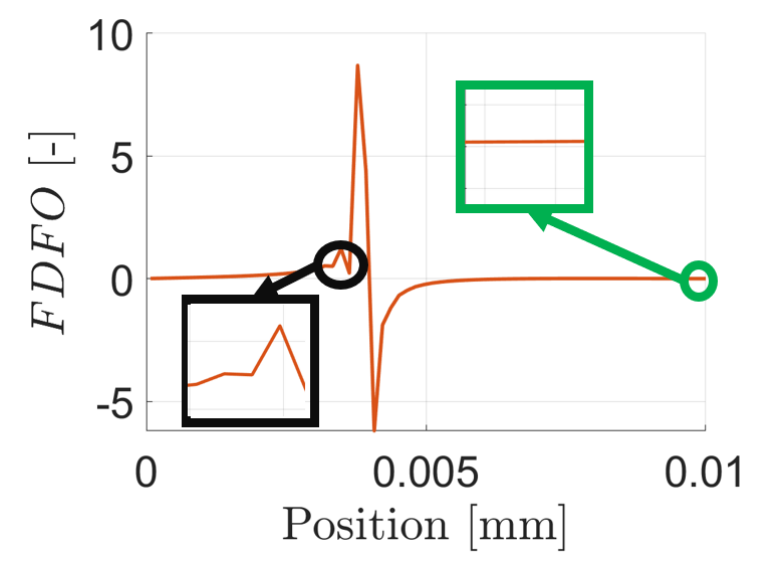

(c)

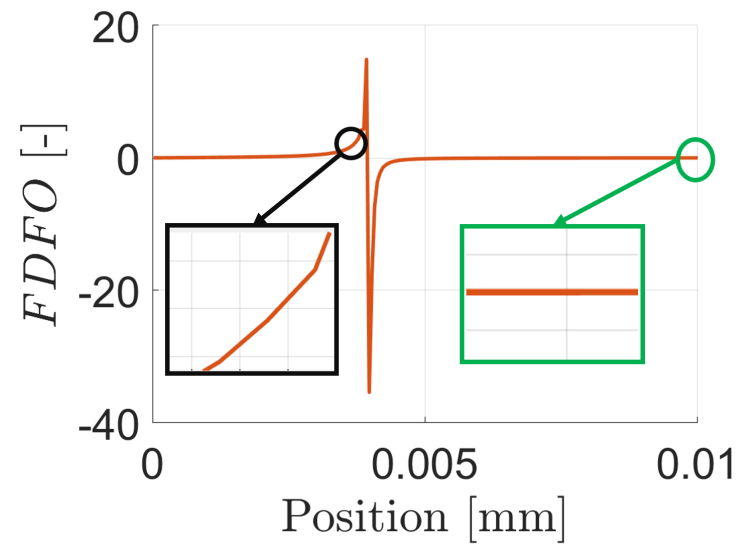

(b)

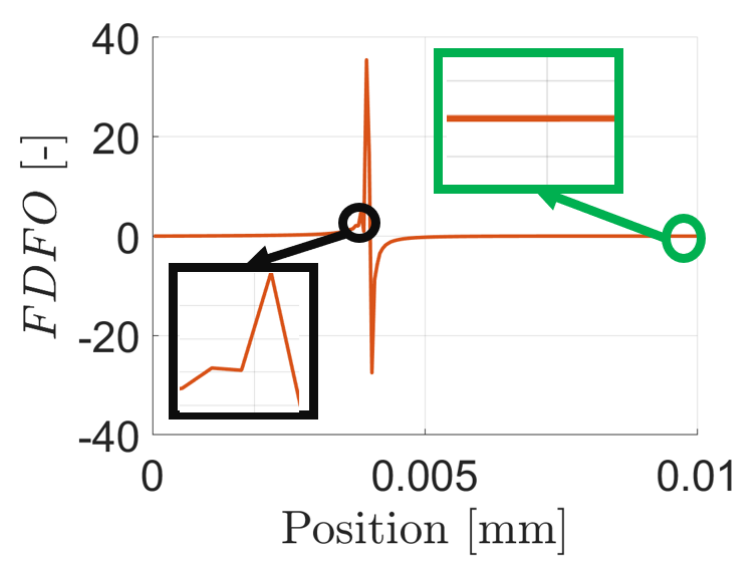

(d)

Figure A.1: model B: The FDFO values of horizontal interface cross line with resolution 135 (a) and resolution 405 (b); The FDFO values of vertical interface cross line with resolution 135 (c) and resolution 405 (d) (Remark: The figure should be viewed in color.)

segment between these two voxels. If a curve is smooth, the slope between neighbour segments should continuously increase or decrease. If some zig-zags are formed in the FDFO curve, the original results have oscillations. As shown in Fig. A.1, the (a) and (b) show the $F D F O$ value of horizontal interface cross line. In these two graphs, non zig-zag can be seen even in views of magnification. Besides the (c) and (d) show FDFO value of vertical interface cross line, where only two oscillations can be seen which are shown in black circles. In general, it can be said that $\boldsymbol{F} \boldsymbol{E} \boldsymbol{M F}$ has almost no oscillation. 


\section{B Appendix B}

Fig. B.1 shows clearly the oscillations of the local strain components along the interface cross section line that occur in models B and C.

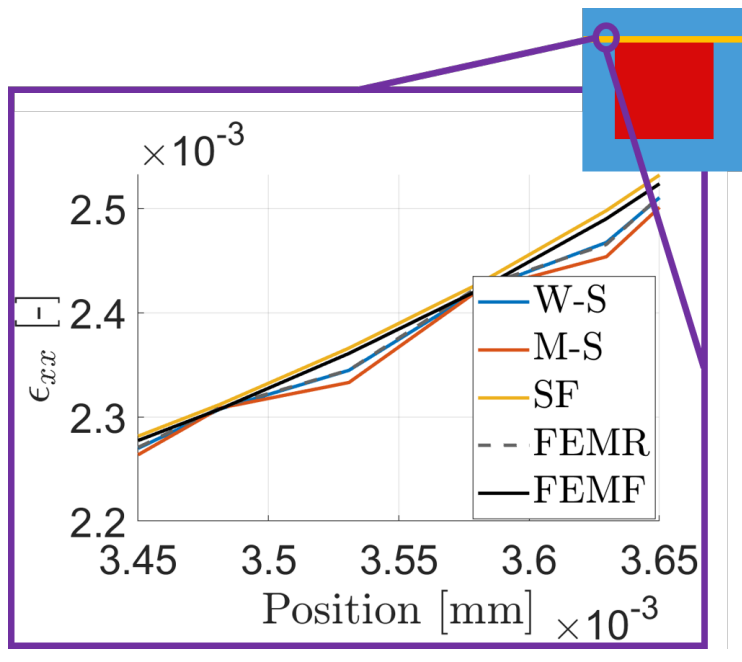

(a)

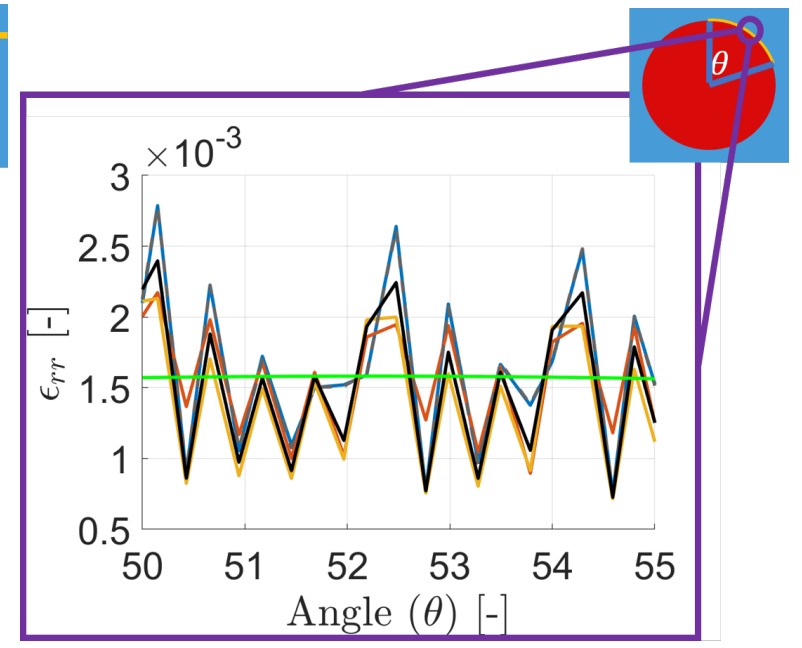

(b)

Figure B.1: (a) describes the $\epsilon_{x x}$ component of model B along the horizontal interface cross section line with $R_{B}=405$; (b) shows the $\epsilon_{r r}$ component of model $\mathrm{C}$ along the interface with $R_{C}=375$. (The green line in (b) is the curve of FEM with conformal mesh)

\section{Appendix C}

As shown in Fig. C.1 (a) and (b) in black circles, the $\boldsymbol{W}-\boldsymbol{S}$ method shows much more oscillations than others at the fiber center which needs a more advanced investigation. 


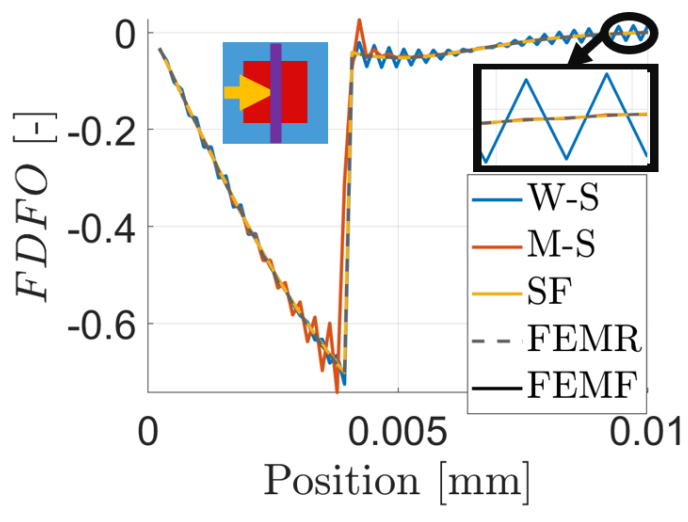

(a)

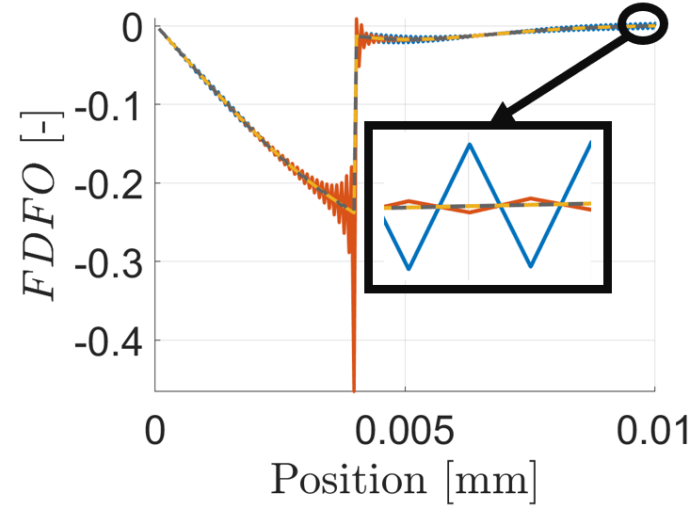

(b)

Figure C.1: model B: (a) The FDFO values of center line with resolution 135 and (b) resolution 405 (Remark: The figure should be viewed in color.)

\section{Appendix D}

Figs. D.1 and D.2 show the relative errors of model C with different FFT algorithms when compared with FEMR and $\boldsymbol{F E M F}$. Because the fiber volume fraction is different for each resolution value, instead of using graphs, all error values are reported in these two figures. 


\begin{tabular}{|c|c|c|c|c|c|c|}
\hline \multicolumn{7}{|c|}{ The maximum error on the interface (Matrix) } \\
\hline \multicolumn{3}{|c|}{ FFTS VS FEMR } & & \multicolumn{3}{|c|}{ FFTs VS FEMF } \\
\hline M-S & SF & W-S & & M-S & $\mathrm{SF}$ & W-S \\
\hline \multicolumn{3}{|c|}{ Res $=225 ; V_{f}=0.5501$} & & \multicolumn{3}{|c|}{ Res $=225 ; V_{f}=0.5501$} \\
\hline $3.48 \mathrm{E}-1$ & $3.49 \mathrm{E}-1$ & $2.24 \mathrm{E}-2$ & & 0.599 & 0.138 & 0.346 \\
\hline \multicolumn{3}{|c|}{ Res $=255 ; V_{f}=0.5499$} & & \multicolumn{3}{|c|}{ Res $=255 ; V_{f}=0.5499$} \\
\hline $3.50 \mathrm{E}-1$ & $3.76 \mathrm{E}-1$ & 3.77E-2 & & 6.29E-1 & $1.44 \mathrm{E}-1$ & $4.27 \mathrm{E}-1$ \\
\hline \multicolumn{3}{|c|}{$\operatorname{Res}=305 ; V_{f}=0.5502$} & & \multicolumn{3}{|c|}{ Res $=305 ; V_{f}=0.5502$} \\
\hline $3.37 \mathrm{E}-1$ & $3.71 \mathrm{E}-1$ & $3.13 \mathrm{E}-2$ & & \begin{tabular}{|l|}
$6.20 \mathrm{E}-1$ \\
\end{tabular} & $1.40 \mathrm{E}-1$ & $4.13 \mathrm{E}-1$ \\
\hline \multicolumn{3}{|c|}{$\operatorname{Res}=345 ; V_{f}=0.5501$} & & \multicolumn{3}{|c|}{ Res $=345 ; V_{f}=0.5501$} \\
\hline $3.46 \mathrm{E}-1$ & $3.55 \mathrm{E}-1$ & $1.65 \mathrm{E}-2$ & & $6.38 \mathrm{E}-1$ & $1.39 \mathrm{E}-1$ & $3.56 \mathrm{E}-1$ \\
\hline \multicolumn{3}{|c|}{ Res $=375 ; V_{f}=0.5497$} & & \multicolumn{3}{|c|}{ Res $=375 ; V_{f}=0.5497$} \\
\hline $3.59 \mathrm{E}-1$ & $3.77 \mathrm{E}-1$ & $4.02 \mathrm{E}-2$ & & 0.666 & 0.140 & 0.431 \\
\hline \multicolumn{3}{|c|}{ Res $=455 ; V_{f}=0.5499$} & & \multicolumn{3}{|c|}{ Res $=455 ; V_{f}=0.5499$} \\
\hline $3.56 \mathrm{E}-1$ & $3.70 \mathrm{E}-1$ & 2.84E-2 & & 6.41E-1 & $1.42 \mathrm{E}-1$ & $4.06 \mathrm{E}-1$ \\
\hline
\end{tabular}

\begin{tabular}{|c|c|c|c|c|c|c|}
\hline \multicolumn{7}{|c|}{ The error of the maximum strain on the interface (Matrix) } \\
\hline \multicolumn{3}{|c|}{ FFTS VS FEMR } & & \multicolumn{3}{|c|}{ FFTs VS FEMF } \\
\hline M-S & SF & W-S & & M-S & SF & W-S \\
\hline \multicolumn{3}{|c|}{ Res $=225 ; V_{f}=0.5501$} & & \multicolumn{3}{|c|}{ Res $=225 ; V_{f}=0.5501$} \\
\hline $2.62 \mathrm{E}-2$ & $4.77 \mathrm{E}-2$ & $1.89 \mathrm{E}-3$ & & 0.0589 & 0.0174 & 0.0338 \\
\hline \multicolumn{3}{|c|}{ Res $=255 ; V_{f}=0.5499$} & & \multicolumn{3}{|c|}{ Res $=255 ; V_{f}=0.5499$} \\
\hline $2.62 \mathrm{E}-2$ & $4.98 \mathrm{E}-2$ & $1.43 \mathrm{E}-3$ & & $6.06 \mathrm{E}-2$ & $1.79 \mathrm{E}-2$ & $3.50 \mathrm{E}-2$ \\
\hline \multicolumn{3}{|c|}{$R e s=305 ; V_{f}=0.5502$} & & \multicolumn{3}{|c|}{$\operatorname{Res}=305 ; V_{f}=0.5502$} \\
\hline $3.52 \mathrm{E}-2$ & $5.03 \mathrm{E}-2$ & $8.20 \mathrm{E}-4$ & & $7.35 \mathrm{E}-2$ & $1.88 \mathrm{E}-2$ & $3.40 \mathrm{E}-2$ \\
\hline \multicolumn{3}{|c|}{ Res $=345 ; V_{f}=0.5501$} & & \multicolumn{3}{|c|}{ Res $=345 ; V_{f}=0.5501$} \\
\hline $2.30 \mathrm{E}-2$ & 4.91E-2 & $1.83 \mathrm{E}-3$ & & $5.67 \mathrm{E}-2$ & $1.80 \mathrm{E}-2$ & $3.47 \mathrm{E}-2$ \\
\hline \multicolumn{3}{|c|}{ Res $=375 ; V_{f}=0.5497$} & & \multicolumn{3}{|c|}{ Res $=375 ; V_{f}=0.5497$} \\
\hline 4.41E-2 & $5.19 \mathrm{E}-2$ & $1.40 \mathrm{E}-4$ & & 0.0834 & 0.0197 & 0.0337 \\
\hline \multicolumn{3}{|c|}{ Res $=455 ; V_{f}=0.5499$} & & \multicolumn{3}{|c|}{ Res $=455 ; V_{f}=0.5499$} \\
\hline $6.67 \mathrm{E}-2$ & $5.01 \mathrm{E}-2$ & $4.96 \mathrm{E}-4$ & & $1.08 \mathrm{E}-1$ & $1.89 \mathrm{E}-2$ & $3.34 \mathrm{E}-2$ \\
\hline
\end{tabular}

Figure D.1: The relative errors of FFTs with FEMs of local strain field $\epsilon_{x x}$ on model C (PART 1)

\section{Declarations}

\section{Funding}

No funds, grants, or other support was received.

\section{Conflicts of interest/Competing interests}

The authors have no conflicts of interest to declare that are relevant to the content of this article. 


\begin{tabular}{|c|c|c|c|c|c|}
\hline \multicolumn{6}{|c|}{ The average error on the interface (Matrix) } \\
\hline \multicolumn{3}{|c|}{ FFTs VS FEMR } & \multicolumn{3}{|c|}{ FFTs VS FEMF } \\
\hline M-S & SF & W-S & M-S & SF & W-S \\
\hline \multicolumn{3}{|c|}{$\operatorname{Res}=225 ; V_{f}=0.5501$} & \multicolumn{3}{|c|}{ Res $=225 ; V_{f}=0.5501$} \\
\hline $8.96 \mathrm{E}-2$ & $1.17 \mathrm{E}-1$ & $6.31 \mathrm{E}-3$ & 0.178 & 0.0509 & 0.0939 \\
\hline \multicolumn{3}{|c|}{ Res $=255 ; V_{f}=0.5499$} & \multicolumn{3}{|c|}{$\operatorname{Res}=255 ; V_{f}=0.5499$} \\
\hline $8.03 \mathrm{E}-2$ & $1.16 \mathrm{E}-1$ & $8.45 \mathrm{E}-3$ & $1.74 \mathrm{E}-1$ & $5.15 \mathrm{E}-2$ & $1.01 \mathrm{E}-1$ \\
\hline \multicolumn{3}{|c|}{$\operatorname{Res}=305 ; V_{f}=0.5502$} & \multicolumn{3}{|c|}{ Res $=305 ; V_{f}=0.5502$} \\
\hline $8.20 \mathrm{E}-2$ & $1.16 \mathrm{E}-1$ & $7.97 \mathrm{E}-3$ & $1.75 \mathrm{E}-1$ & $5.08 \mathrm{E}-2$ & $9.83 \mathrm{E}-2$ \\
\hline \multicolumn{3}{|c|}{$\operatorname{Res}=345 ; V_{f}=0.5501$} & \multicolumn{3}{|c|}{$\operatorname{Res}=345 ; V_{f}=0.5501$} \\
\hline $8.96 \mathrm{E}-2$ & $1.16 \mathrm{E}-1$ & $5.51 \mathrm{E}-3$ & $1.78 \mathrm{E}-1$ & $5.09 \mathrm{E}-2$ & $9.27 \mathrm{E}-2$ \\
\hline \multicolumn{3}{|c|}{$\operatorname{Res}=375 ; V_{f}=0.5497$} & \multicolumn{3}{|c|}{$\operatorname{Res}=375 ; V_{f}=0.5497$} \\
\hline $7.91 \mathrm{E}-2$ & $1.16 \mathrm{E}-2$ & $7.15 \mathrm{E}-3$ & 0.173 & 0.0513 & 0.0983 \\
\hline \multicolumn{3}{|c|}{ Res $=455 ; V_{f}=0.5499$} & \multicolumn{3}{|c|}{ Res $=455 ; V_{f}=0.5499$} \\
\hline $8.50 \mathrm{E}-2$ & $1.17 \mathrm{E}-1$ & $6.72 \mathrm{E}-3$ & $1.75 \mathrm{E}-1$ & $5.12 \mathrm{E}-2$ & $9.45 \mathrm{E}-2$ \\
\hline
\end{tabular}

\begin{tabular}{|c|c|c|c|c|c|}
\hline \multicolumn{6}{|c|}{ The average error at the fiber center (Fiber) } \\
\hline \multicolumn{3}{|c|}{ FFTs VS FEMR } & \multicolumn{3}{|c|}{ FFTs VS FEMF } \\
\hline M-S & SF & W-S & M-S & SF & W-S \\
\hline \multicolumn{3}{|c|}{ Res $=225 ; V_{f}=0.5501$} & \multicolumn{3}{|c|}{ Res $=225 ; V_{f}=0.5501$} \\
\hline $3.73 \mathrm{E}-3$ & $1.57 \mathrm{E}-3$ & $8.82 \mathrm{E}-4$ & 0.00440 & 0.000893 & 0.000908 \\
\hline \multicolumn{3}{|c|}{ Res $=255 ; V_{f}=0.5499$} & \multicolumn{3}{|c|}{ Res $=255 ; V_{f}=0.5499$} \\
\hline $3.02 \mathrm{E}-3$ & $1.22 \mathrm{E}-3$ & $7.60 \mathrm{E}-4$ & $3.54 \mathrm{E}-3$ & $6.98 \mathrm{E}-4$ & $7.80 \mathrm{E}-4$ \\
\hline \multicolumn{3}{|c|}{ Res $=305 ; V_{f}=0.5502$} & \multicolumn{3}{|c|}{ Res $=305 ; V_{f}=0.5502$} \\
\hline $2.34 \mathrm{E}-3$ & $1.06 \mathrm{E}-3$ & $1.15 \mathrm{E}-4$ & $2.82 \mathrm{E}-3$ & $5.80 \mathrm{E}-4$ & $5.36 \mathrm{E}-4$ \\
\hline \multicolumn{3}{|c|}{ Res $=345 ; V_{f}=0.5501$} & \multicolumn{3}{|c|}{$\operatorname{Res}=345 ; V_{f}=0.5501$} \\
\hline $2.31 \mathrm{E}-3$ & $9.31 \mathrm{E}-4$ & $1.25 \mathrm{E}-4$ & $2.71 \mathrm{E}-3$ & $5.25 \mathrm{E}-4$ & $4.33 \mathrm{E}-4$ \\
\hline \multicolumn{3}{|c|}{ Res $=375 ; V_{f}=0.5497$} & \multicolumn{3}{|c|}{$\operatorname{Res}=375 ; V_{f}=0.5497$} \\
\hline $2.00 \mathrm{E}-3$ & $8.06 \mathrm{E}-4$ & $3.66 \mathrm{E}-4$ & 0.00235 & 0.000461 & 0.000364 \\
\hline \multicolumn{3}{|c|}{ Res $=455 ; V_{f}=0.5499$} & \multicolumn{3}{|c|}{$R e s=455 ; V_{f}=0.5499$} \\
\hline $1.76 \mathrm{E}-3$ & $6.61 \mathrm{E}-4$ & $6.84 \mathrm{E}-5$ & $2.06 \mathrm{E}-3$ & $3.55 \mathrm{E}-4$ & $3.75 \mathrm{E}-4$ \\
\hline
\end{tabular}

\begin{tabular}{|c|c|c|c|c|c|c|}
\hline \multicolumn{7}{|c|}{ The average error in the volume } \\
\hline \multicolumn{3}{|c|}{ FFTs VS FEMR } & & \multicolumn{3}{|c|}{ FFTs VS FEMF } \\
\hline M-S & SF & W-S & & M-S & SF & W-S \\
\hline \multicolumn{3}{|c|}{$R e s=225 ; V_{f}=0.5501$} & & \multicolumn{3}{|c|}{$R e s=225 ; V_{f}=0.5501$} \\
\hline $1.30 \mathrm{E}-2$ & $9.89 \mathrm{E}-3$ & $1.60 \mathrm{E}-3$ & & 0.0148 & 0.00347 & 0.00747 \\
\hline \multicolumn{3}{|c|}{$R e s=255 ; V_{f}=0.5499$} & & \multicolumn{3}{|c|}{ Res $=255 ; V_{f}=0.5499$} \\
\hline $1.10 \mathrm{E}-2$ & $8.91 \mathrm{E}-3$ & $1.65 \mathrm{E}-3$ & & $1.28 \mathrm{E}-2$ & $3.05 \mathrm{E}-3$ & $6.85 \mathrm{E}-3$ \\
\hline \multicolumn{3}{|c|}{ Res $=305 ; V_{f}=0.5502$} & & \multicolumn{3}{|c|}{ Res $=305 ; V_{f}=0.5502$} \\
\hline $9.69 \mathrm{E}-3$ & $7.40 \mathrm{E}-3$ & $1.47 \mathrm{E}-3$ & & $1.11 \mathrm{E}-2$ & $2.56 \mathrm{E}-3$ & $5.80 \mathrm{E}-3$ \\
\hline \multicolumn{3}{|c|}{$\operatorname{Res}=345 ; V_{f}=0.5501$} & & \multicolumn{3}{|c|}{ Res $=345 ; V_{f}=0.5501$} \\
\hline $8.83 \mathrm{E}-3$ & $6.65 \mathrm{E}-3$ & $8.68 \mathrm{E}-4$ & & $1.00 \mathrm{E}-2$ & $2.28 \mathrm{E}-3$ & $5.09 \mathrm{E}-3$ \\
\hline \multicolumn{3}{|c|}{$\operatorname{Res}=375 ; V_{f}=0.5497$} & & \multicolumn{3}{|c|}{ Res $=375 ; V_{f}=0.5497$} \\
\hline 0.00786 & 0.00620 & 0.00107 & & 0.00906 & 0.00210 & 0.00477 \\
\hline \multicolumn{3}{|c|}{ Res $=455 ; V_{f}=0.5499$} & & \multicolumn{3}{|c|}{ Res $=455 ; V_{f}=0.5499$} \\
\hline $6.95 \mathrm{E}-3$ & $5.14 \mathrm{E}-3$ & $7.93 \mathrm{E}-4$ & & $7.90 \mathrm{E}-3$ & $1.73 \mathrm{E}-3$ & $3.92 \mathrm{E}-3$ \\
\hline
\end{tabular}

Figure D.2: The relative errors of FFTs with FEMs of local strain field $\epsilon_{x x}$ on model C (PART 2) 


\section{Availability of data and material}

The datasets generated during and/or analysed during the current study are available from the corresponding author on reasonable request.

\section{Code availability}

The code used for the current study is available from the corresponding author on reasonable request.

\section{References}

[1] S. V. Lomov, D. S. Ivanov, I. Verpoest, M. Zako, T. Kurashiki, H. Nakai, and S. Hirosawa, "Meso-FE modelling of textile composites: Road map, data flow and algorithms," Composites Science and Technology, vol. 67, no. 9, pp. 1870-1891, 2007.

[2] J. J. Espadas-Escalante, B. A. Bednarcyk, E. J. Pineda, and P. Isaksson, "Modeling the influence of layer shifting on the properties and nonlinear response of woven composites subject to continuum damage," Composite Structures, vol. 220, pp. 539549, 72019 .

[3] M. Herráez, D. Mora, F. Naya, C. S. Lopes, C. González, and J. Llorca, "Transverse cracking of cross-ply laminates: A computational micromechanics perspective," Composites Science and Technology, vol. 110, pp. 196-204, 42015.

[4] G. Grail, M. Hirsekorn, A. Wendling, G. Hivet, and R. Hambli, "Consistent Finite Element mesh generation for meso-scale modeling of textile composites with preformed and compacted reinforcements," Composites Part A: Applied Science and Manufacturing, vol. 55, pp. 143-151, 2013.

[5] B. Wintiba, B. Sonon, K. Ehab Moustafa Kamel, and T. J. Massart, "An automated procedure for the generation and conformal discretization of $3 \mathrm{D}$ woven composites RVEs," Composite Structures, vol. 180, pp. 955-971, 112017. 
[6] W. G. Jiang, S. R. Hallett, and M. R. Wisnom, "Development of Domain Superposition Technique for the modelling of woven fabric composites," in Computational Methods in Applied Sciences, vol. 10, pp. 281-291, Springer Netherland, 2008.

[7] J. Gao, W. Chen, B. Yu, P. Fan, B. Zhao, J. Hu, D. Zhang, G. Fang, and F. Peng, "A multi-scale method for predicting ABD stiffness matrix of single-ply weave-reinforced composite," Composite Structures, vol. 230, p. 111478, 122019.

[8] S. A. Tabatabaei, S. V. Lomov, and I. Verpoest, "Assessment of embedded element technique in meso-FE modelling of fibre reinforced composites," Composite Structures, vol. 107, pp. 436-446, 12014.

[9] O. Vorobiov, S. A. Tabatabaei, and S. V. Lomov, "Mesh superposition applied to meso-FE modelling of fibre-reinforced composites: Cross-comparison of implementations," International Journal for Numerical Methods in Engineering, vol. 111, pp. 1003-1024, 92017.

[10] S. D. Green, M. Y. Matveev, A. C. Long, D. Ivanov, and S. R. Hallett, "Mechanical modelling of 3D woven composites considering realistic unit cell geometry," Composite Structures, vol. 118, no. 1, pp. 284-293, 2014.

[11] S. Yan, X. Zeng, and A. Long, "Meso-scale modelling of 3D woven composite T-joints with weave variations," Composites Science and Technology, vol. 171, pp. 171-179, 22019.

[12] G. Ernst, M. Vogler, C. Hühne, and R. Rolfes, "Multiscale progressive failure analysis of textile composites," Composites Science and Technology, vol. 70, no. 1, pp. 61-72, 2010.

[13] S. Yu, D. Zhang, and K. Qian, "Numerical Analysis of Macro-Scale Mechanical Behaviors of 3D Orthogonal Woven Composites using a Voxel-Based Finite Element Model," Applied Composite Materials, vol. 26, no. 1, pp. 65-83, 2019.

[14] Y. Liu, I. Straumit, D. Vasiukov, S. V. Lomov, and S. Panier, "Prediction of linear and non-linear behavior of 3D woven composite using mesoscopic voxel mod- 
els reconstructed from X-ray micro-tomography," Composite Structures, vol. 179, pp. 568-579, 112017.

[15] F. Montero-Chacón, J. Marín-Montín, and F. Medina, "Mesomechanical characterization of porosity in cementitious composites by means of a voxel-based finite element model," Computational Materials Science, vol. 90, pp. 157-170, 72014.

[16] A. Doitrand, C. Fagiano, F. X. Irisarri, and M. Hirsekorn, "Comparison between voxel and consistent meso-scale models of woven composites," Composites Part A: Applied Science and Manufacturing, vol. 73, pp. 143-154, 2015.

[17] B. Butrylo, F. Musy, L. Nicolas, R. Perrussel, R. Scorretti, and C. Vollaire, "A survey of parallel solvers for the finite element method in computational electromagnetics," COMPEL - The International Journal for Computation and Mathematics in Electrical and Electronic Engineering, vol. 23, pp. 531-546, 62004.

[18] Y. Zhu, E. Sifakis, J. Teran, and A. Brandt, "An efficient multigrid method for the simulation of high-resolution elastic solids," ACM Transactions on Graphics, vol. 29, pp. 1-18, 32010 .

[19] Y. Chen, L. Gélébart, C. Chateau, M. Bornert, C. Sauder, and A. King, "Analysis of the damage initiation in a $\mathrm{SiC} / \mathrm{SiC}$ composite tube from a direct comparison between large-scale numerical simulation and synchrotron X-ray micro-computed tomography," International Journal of Solids and Structures, vol. 161, pp. 111-126, 2019.

[20] H. Moulinec and P. Suquet, "A fast numerical method for computing the linear and nonlinear mechanical properties of composites," Comptes rendus de l'Académie des sciences. Série II, Mécanique, physique, chimie, astronomie, vol. 318, no. 11, pp. 1417-1423, 1994.

[21] H. Moulinec and P. Suquet, "A FFT-Based Numerical Method for Computing the Mechanical Properties of Composites from Images of their Microstructures," in $I U$ TAM Symposium on Microstructure-Property Interactions in Composite Materials, pp. 235-246, Springer Netherlands, 1995. 
[22] H. Moulinec and P. Suquet, "A numerical method for computing the overall response of nonlinear composites with complex microstructure," Computer Methods in Applied Mechanics and Engineering, vol. 157, pp. 69-94, 41998.

[23] S. Liu, X. Jin, Z. Wang, L. M. Keer, and Q. Wang, "Analytical solution for elastic fields caused by eigenstrains in a half-space and numerical implementation based on fft," International Journal of Plasticity, vol. 35, pp. 135 - 154, 2012.

[24] Z. Wang, H. Yu, and Q. Wang, "Analytical solutions for elastic fields caused by eigenstrains in two joined and perfectly bonded half-spaces and related problems," International Journal of Plasticity, vol. 76, pp. 1-28, 82016.

[25] R. A. Lebensohn, A. K. Kanjarla, and P. Eisenlohr, "An elasto-viscoplastic formulation based on fast Fourier transforms for the prediction of micromechanical fields in polycrystalline materials," International Journal of Plasticity, vol. 32-33, pp. 59-69, 2012.

[26] R. Ma and T. J. Truster, "FFT-based homogenization of hypoelastic plasticity at finite strains," Computer Methods in Applied Mechanics and Engineering, vol. 349, pp. 499-521, 62019.

[27] P. Suquet, H. Moulinec, O. Castelnau, M. Montagnat, N. Lahellec, F. Grennerat, P. Duval, and R. Brenner, "Multi-scale modeling of the mechanical behavior of polycrystalline ice under transient creep," in Procedia IUTAM, vol. 3, pp. 76-90, Elsevier B.V., 52012.

[28] T. Otsuka, R. Brenner, and B. Bacroix, "FFT-based modelling of transformation plasticity in polycrystalline materials during diffusive phase transformation," International Journal of Engineering Science, vol. 127, pp. 92-113, 62018.

[29] C. Paramatmuni and A. K. Kanjarla, "A crystal plasticity FFT based study of deformation twinning, anisotropy and micromechanics in HCP materials: Application to AZ31 alloy," International Journal of Plasticity, vol. 113, pp. 269-290, 22019.

[30] S. El Shawish, P. G. Vincent, H. Moulinec, L. Cizelj, and L. Gélébart, "Full-field polycrystal plasticity simulations of neutron-irradiated austenitic stainless steel: A 
comparison between FE and FFT-based approaches," Journal of Nuclear Materials, vol. 529, p. 151927, 22020.

[31] M. Li, Y. Cao, W. Shen, and J. Shao, "A damage model of mechanical behavior of porous materials: Application to sandstone," International Journal of Damage Mechanics, vol. 27, pp. 1325-1351, 92018.

[32] Y. Cao, W. Shen, J. Shao, and W. Wang, "A novel FFT-based phase field model for damage and cracking behavior of heterogeneous materials," International Journal of Plasticity, vol. 133, p. 102786, 102020.

[33] W. Leclerc, N. Ferguen, C. Pélegris, H. Haddad, E. Bellenger, and M. Guessasma, "A numerical investigation of effective thermoelastic properties of interconnected alumina/Al composites using FFT and FE approaches," Mechanics of Materials, vol. 92, pp. 42-57, 12016.

[34] V. Monchiet, "FFT based iterative schemes for composites conductors with nonoverlapping fibers and Kapitza interface resistance," International Journal of Solids and Structures, vol. 135, pp. 14-25, 32018.

[35] S. Staub, H. Andrä, and M. Kabel, "Fast FFT based solver for rate-dependent deformations of composites and nonwovens," International Journal of Solids and Structures, vol. 154, pp. 33-42, 122018.

[36] B. Wang, G. Fang, S. Liu, and J. Liang, "Effect of heterogeneous interphase on the mechanical properties of unidirectional fiber composites studied by FFT-based method," Composite Structures, vol. 20, pp. 642 - 651, 2019.

[37] J. Li, S. Meng, X. Tian, F. Song, and C. Jiang, "A non-local fracture model for composite laminates and numerical simulations by using the FFT method," Composites Part B: Engineering, vol. 43, no. 3, pp. 961-971, 2012.

[38] B. Wang, G. Fang, S. Liu, M. Fu, and J. Liang, "Progressive damage analysis of 3D braided composites using FFT-based method," Composite Structures, vol. 192, no. 2, pp. 255-263, 2018. 
[39] G. Fang, B. Wang, and J. Liang, "A coupled FE-FFT multiscale method for progressive damage analysis of 3D braided composite beam under bending load," Composites Science and Technology, vol. 181, 92019.

[40] D. J. Eyre and G. W. Milton, "A fast numerical scheme for computing the response of composites using grid refinement," The European Physical Journal Applied Physics, vol. 6, pp. 41-47, 41999.

[41] J. C. Michel, H. Moulinec, and P. Suquet, "A computational method based on augmented lagrangians and fast fourier transforms for composites with high contrast," Computer Modeling in Engineering and Sciences, vol. 1, no. 2, pp. 79-88, 2000.

[42] V. Monchiet and G. Bonnet, "A polarization-based FFT iterative scheme for computing the effective properties of elastic composites with arbitrary contrast," International Journal for Numerical Methods in Engineering, vol. 89, pp. 1419-1436, 3 2012.

[43] S. Brisard and L. Dormieux, "FFT-based methods for the mechanics of composites: A general variational framework," Computational Materials Science, vol. 49, no. 3, pp. 663-671, 2010.

[44] J. Zeman, J. Vondřejc, J. Novák, and I. Marek, "Accelerating a FFT-based solver for numerical homogenization of periodic media by conjugate gradients," Journal of Computational Physics, vol. 229, pp. 8065-8071, 102010.

[45] L. Gélébart and R. Mondon-Cancel, "Non-linear extension of FFT-based methods accelerated by conjugate gradients to evaluate the mechanical behavior of composite materials," Computational Materials Science, vol. 77, pp. 430-439, 92013.

[46] K. S. Eloh, A. Jacques, and S. Berbenni, "Development of a new consistent discrete green operator for FFT-based methods to solve heterogeneous problems with eigenstrains," International Journal of Plasticity, vol. 116, pp. 1-23, 52019.

[47] M. Leuschner and F. Fritzen, "Fourier-Accelerated Nodal Solvers (FANS) for homogenization problems," Computational Mechanics, vol. 62, pp. 359-392, 2018. 
[48] S. Kaßbohm, W. H. Müller, and R. Feßler, "Improved approximations of Fourier coefficients for computing periodic structures with arbitrary stiffness distribution," Computational Materials Science, vol. 37, pp. 90-93, 82006.

[49] P. Shanthraj, P. Eisenlohr, M. Diehl, and F. Roters, "Numerically robust spectral methods for crystal plasticity simulations of heterogeneous materials," International Journal of Plasticity, vol. 66, pp. 31-45, 32015.

[50] S. Brisard and L. Dormieux, "Combining Galerkin approximation techniques with the principle of Hashin and Shtrikman to derive a new FFT-based numerical method for the homogenization of composites," Computer Methods in Applied Mechanics and Engineering, vol. 217-220, pp. 197-212, 42012.

[51] W. Müller, "Mathematical vs. Experimental Stress Analysis of Inhomogeneities in Solids," in International Seminar on Mechanics and Mechanisms of Solid-Solid Phase Transformations, vol. 06, pp. 139-148, Springer, Berlin, Heidelberg, 1996.

[52] W. Dreyer, W. H. Müller, and J. Olschewski, "Approximate analytical 2D-solution for the stresses and strains in eigenstrained cubic materials," Acta Mechanica, vol. 136, no. 3, pp. 171-192, 1999.

[53] C. M. Brown, W. Dreyer, and W. H. Müller, "Discrete fourier transforms and their application to stress-strain problems in composite mechanics: A convergence study," Proceedings of the Royal Society A: Mathematical, Physical and Engineering Sciences, vol. 458, pp. 1967-1987, 82002.

[54] F. Willot and Y.-P. Pellegrini, "Fast Fourier Transform computations and build-up of plastic deformation in 2D, elastic-perfectly plastic, pixelwise disordered porous media," in 11th International Symposium on Continuum Models and Discrete Systems, pp. 443-449, Presses des mines, 72008.

[55] F. Willot, B. Abdallah, and Y.-P. Pellegrini, "Fourier-based schemes with modified Green operator for computing the electrical response of heterogeneous media with accurate local fields," International Journal for Numerical Methods in Engineering, vol. 98, pp. 518-533, 52014. 
[56] S. Berbenni, V. Taupin, K. S. Djaka, and C. Fressengeas, "A numerical spectral approach for solving elasto-static field dislocation and g-disclination mechanics," International Journal of Solids and Structures, vol. 51, pp. 4157-4175, 112014.

[57] R. A. Lebensohn and A. Needleman, "Numerical implementation of non-local polycrystal plasticity using fast Fourier transforms," Journal of the Mechanics and Physics of Solids, vol. 97, pp. 333-351, 122016.

[58] K. S. Djaka, A. Villani, V. Taupin, L. Capolungo, and S. Berbenni, "Field Dislocation Mechanics for heterogeneous elastic materials: A numerical spectral approach," Computer Methods in Applied Mechanics and Engineering, vol. 315, pp. 921-942, 3 2017.

[59] A. Vidyasagar, W. L. Tan, and D. M. Kochmann, "Predicting the effective response of bulk polycrystalline ferroelectric ceramics via improved spectral phase field methods," Journal of the Mechanics and Physics of Solids, vol. 106, pp. 133-151, 92017.

[60] F. Willot, "Fourier-based schemes for computing the mechanical response of composites with accurate local fields," Comptes Rendus - Mecanique, vol. 343, no. 3, pp. 232-245, 2015.

[61] "AMITEX-FFTP." http://www.maisondelasimulation.fr/projects/amitex/general/_build/html/.

[62] M. Schneider, D. Merkert, and M. Kabel, "FFT-based homogenization for microstructures discretized by linear hexahedral elements," International Journal for Numerical Methods in Engineering, vol. 109, pp. 1461-1489, 32017.

[63] M. Kabel, D. Merkert, and M. Schneider, "Use of composite voxels in FFT-based homogenization," Computer Methods in Applied Mechanics and Engineering, vol. 294, pp. 168-188, 2015.

[64] R. Charière, A. Marano, and L. Gélébart, "Use of composite voxels in FFT based elastic simulations of hollow glass microspheres/polypropylene composites," International Journal of Solids and Structures, vol. 182-183, pp. 1-14, 12020. 
[65] X. Ma, K. K. Parvathaneni, S. Lomov, D. Vasiukov, M. Shakoor, and C. H. Park, "Quantitative comparison between fast fourier transform and finite element method for micromechanical modeling of composite," in FiBreMOD conference 2019, 12 2019.

[66] G. Fang, B. El Said, D. Ivanov, and S. R. Hallett, "Smoothing artificial stress concentrations in voxel-based models of textile composites," Composites Part A: Applied Science and Manufacturing, vol. 80, pp. 270-284, 2016.

[67] S. G. Abaimov, A. A. Khudyakova, and S. V. Lomov, "On the closed form expression of the Mori-Tanaka theory prediction for the engineering constants of a unidirectional fiber-reinforced ply," Composite Structures, vol. 142, pp. 1-6, 52016.

[68] M. Kabel, S. Fliegener, and M. Schneider, "Mixed boundary conditions for FFTbased homogenization at finite strains," Computational Mechanics, vol. 57, no. 2, pp. 193-210, 2016. 